\title{
Minimally unstable Pareto improvements over deferred acceptance
}

\author{
BATtAL DoĞAN \\ Department of Economics, University of Bristol
}

\author{
LARS EHLERS \\ Département de Sciences Économiques and CIREQ, Université de Montréal
}

\begin{abstract}
We investigate efficient and minimally unstable Pareto improvements over the deferred acceptance (DA) mechanism-a popular school choice mechanism that is stable but not efficient. We show that there is no Pareto improvement over the DA mechanism that is minimally unstable among efficient assignments when the stability comparison is based on counting the number of blocking pairs. Our main result characterizes the priority profiles for which there exists a Pareto improvement over the DA assignment that is minimally unstable among efficient assignments. We further consider an alternative natural stability comparison based on the set of blocking students who are involved in at least one blocking pair, show that the impossibilities remain, and characterize the possibility domain of priority profiles.
\end{abstract}

KeYwords. School choice, deferred acceptance, stability comparisons, cardinal minimal instability.

JEL Classification. C70, D47, D61, D63.

\section{INTRODUCTION}

Assigning students to schools in a desirable way, taking into account both the preferences of the students and their priorities at different schools, has led to an extensive school choice literature starting with the seminal study by Abdulkadiroğlu and Sönmez (2003). In the meantime, centralized school choice systems have been implemented in many school districts around the world. Both in theory and in practice, the deferred acceptance (DA) mechanism (Abdulkadiroğlu and Sönmez (2003)), which is based on the DA algorithm (Gale and Shapley (1962)), stands out as a central school choice mechanism.

Battal Doğan: battal dogan@bristol.ac.uk

Lars Ehlers: lars.ehlers@umontreal.ca

This article is a substantial revision of the formerly titled paper "Blocking Pairs versus Blocking Students: Stability Comparisons in School Choice” (Doğan and Ehlers (2020a)). Battal Doğan acknowledges financial support from the British Academy/Leverhulme Trust (SRG1819\190133). Lars Ehlers acknowledges financial support from the SSHRC (Canada) and the FRQSC (Québec). We are grateful to two anonymous referees for their useful comments and suggestions. We thank seminar participants at the University of Oxford and participants of the 39th Bosphorus Workshop on Economic Design and the Lisbon Meetings in Game Theory and Applications for valuable comments.

(C) 2021 The Authors. Licensed under the Creative Commons Attribution-NonCommercial License 4.0. Available at https://econtheory.org. https://doi.org/10.3982/TE4257 
The DA mechanism offers a compromise between two natural and desirable properties that are, in general, incompatible: (Pareto) efficiency and stability. An assignment is efficient if there is no other assignment at which a student is better off while no student is worse off. An assignment is stable (or fair) if it does not involve a "blocking pair" of a student and a school such that the student prefers the school to his assigned school and he has a higher priority than another student who is assigned to that school. Unfortunately, there are school choice problems without an assignment that is both efficient and stable. ${ }^{1}$ The DA mechanism is stable, and although it is not efficient, it is "constrained efficient" as it always chooses the students-optimal (with respect to the Pareto dominance comparison) stable assignment (Gale and Shapley (1962)).

Given the inefficiency of the DA mechanism, the literature has been investigating Pareto improvements over the DA mechanism. ${ }^{2}$ In this paper, we insist on efficiency and investigate Pareto improvements over the DA assignment that are minimally unstable among efficient assignments: such assignments are efficient and there is no other efficient assignment that is more stable. To formulate what it means to be more stable, we first consider comparing assignments by comparing their sets of blocking (studentschool) pairs. An assignment is more stable than another assignment if the set of blocking pairs in the former is a proper subset of the set of blocking pairs in the latter assignment. This method has a corresponding cardinal version where an assignment is cardinally more stable than another assignment if the number of blocking pairs in the former assignment is less than the number of blocking pairs in the latter assignment. Minimal instability is a relatively weak requirement as many assignments are incomparable when their sets of blocking pairs are compared in the set inclusion sense, which is not the case for cardinal minimal instability. ${ }^{3}$

It turns out that there is a Pareto improvement over the DA mechanism that is minimally unstable among efficient assignments: the efficiency adjusted deferred acceptance (EADA) mechanism (Kesten (2010)). That is, at each problem, the EADA mechanism produces an assignment that is minimally unstable among efficient assignments. ${ }^{4}$ However, there is no Pareto improvement over the DA mechanism that is cardinally minimally unstable among efficient assignments (Proposition 2). Our main result characterizes the priority profiles for which there exists a Pareto improvement over the DA assignment that is cardinally minimally unstable among efficient assignments (Theorem 1). ${ }^{5}$ Our characterization result provides two important insights.

\footnotetext{
${ }^{1}$ This follows from an example in Roth (1982). It is more explicitly shown in Abdulkadiroğlu and Sönmez (2003).

${ }^{2}$ See, among others, Abdulkadiroğlu et al. (2015), Che and Tercieux (2019), Kesten (2010), Dur et al. (2019), Erdil and Ergin (2008), and Kesten and Kurino (2019). Pareto improvements over the DA mechanism violate strategy-proofness (Abdulkadiroğlu et al. (2009), Kesten (2010), Kesten and Kurino (2019)). We discuss strategy-proofness in Section 6.

${ }^{3}$ Clearly, if an assignment is more stable than another assignment, then it is also cardinally more stable.

${ }^{4}$ This result also follows from Tang and Zhang (2021). We present our proof, which is independent and different.

${ }^{5}$ The main result is presented in the unit capacity setup. In Appendix A.2, we show that the characterizing conditions are still necessary conditions for the EADA assignment to be cardinally minimally unstable among efficient assignments in the general multicapacity case and essentially the main insights extend to the general setup.
} 
(a) For any priority profile for which there exists a Pareto improvement over the DA assignment that is cardinally minimally unstable among efficient assignments, the DA assignment always includes at most one improvement cycle ${ }^{6}$ and there is a unique efficient Pareto improvement over the DA assignment. This result suggests that such priority profiles are quite restricted.

(b) Our characterization result fully uncovers the three potential reasons why a Pareto improvement over the DA assignment, for example, the EADA assignment, may fail to be cardinally minimally unstable among efficient assignments. Each potential reason corresponds to the violation of one of the three conditions in Theorem 1. A key condition is based on a novel acyclicity notion.

Comparing the sets of blocking pairs is a reasonable stability comparison method in the context of school choice because a student who is involved in a blocking pair is being treated unfairly, which makes the assignment open to criticism on fairness grounds and even on legal grounds, since the student may pursue legal action against the school district. ${ }^{7}$ Minimizing blocking pairs, in a sense, minimizes unfairness and possibilities of legal action. The same perspective justifies another natural stability comparison: comparing the sets of blocking students, i.e., students involved in at least one blocking pair. According to this alternative method, an assignment is blocking-student-wise (BS-wise) more stable than another assignment if the set of blocking students in the former is a proper subset of the set of blocking students in the latter assignment. This method also has a corresponding cardinal version such that an assignment is BS-wise cardinally more stable than another assignment if the number of blocking students in the former is less than the number of blocking students in the latter assignment.

It turns out that there is no implication relation between any of the blockingstudent-wise notions and any of the blocking-pair-wise notions. More interestingly, there is no mechanism that Pareto improves over the DA mechanism and that is BSwise minimally unstable among efficient assignments (Proposition 3), and, in particular, the EADA mechanism is not BS-wise minimally unstable among efficient assignments. Our results suggest that the EADA mechanism's failure of cardinal minimal instability while satisfying minimal instability cannot be solely attributed to the cardinal feature of the comparison method and that several conclusions related to minimal instability are sensitive to the choice of the stability comparison method. ${ }^{8}$ We characterize the priority profiles for which there exists a Pareto improvement over the DA assignment that is BS-wise cardinally minimally unstable among efficient assignments (Theorem 2), which results in similar insights as above: For any "possibility priority profile," there is a unique efficient Pareto improvement over the DA assignment, which includes exactly one blocking student, and our characterization result again fully uncovers all potential reasons for impossibility.

\footnotetext{
${ }^{6}$ See Section 3 for a definition of an improvement cycle.

${ }^{7}$ Ehlers and Morrill (2020) provide a thorough analysis of legal assignments in school choice.

${ }^{8}$ Not all conclusions have to be sensitive, however. In Doğan and Ehlers (2020b), we show that a result by Abdulkadiroğlu et al. (2020), which relies on a particular stability comparison method, is, in fact, robust to the choice of the stability comparison method.
} 


\subsection{Related literature}

Our methods to compare assignments by their stability are inspired by comparison methods in recent studies (here, we provide a non-exhaustive list). In Pathak and Sönmez (2013), school choice mechanisms are compared in terms of their manipulability by comparing the sets of problems, in the set inclusion sense, at which they are manipulable. In Andersson et al. (2014), resource allocation mechanisms (in the model of allocating objects with monetary transfers) are compared also in terms of their manipulability, but by comparing cardinalities of the sets of problems at which they are manipulable. Similar comparison methods have been used to compare manipulability of social choice functions (Maus et al. (2007a, 2007b)) and the efficiency of probabilistic assignments (Doğan et al. (2018)). Although our study uses similar comparison methods, we depart from these studies by focusing on stability. While stability comparisons in school choice have been studied only recently, there is an earlier literature in the context of roommates problems. Among others, Abraham et al. (2005) define almost stable matchings as matchings that minimize the number of blocking pairs, which is the roommates problem counterpart of the blocking-pairs and blocking-students cardinality comparisons considered in our paper.

Abdulkadiroğlu et al. (2020), ${ }^{9}$ Kwon and Shorrer (2019), and Tang and Zhang (2021) also compare school choice mechanisms, and assignments, in terms of their stability. Although these studies also consider minimally unstable assignments, to our knowledge, our study is the first to consider cardinally minimally unstable assignments and blocking-student-wise comparisons in school choice. Tang and Zhang (2021) introduce the notion of self-constrained optimality for assignments, which requires that the assignment Pareto dominates any other assignment that is more stable, and show that the EADA assignment is self-constrained optimal at each problem. This result also implies that the EADA mechanism is minimally unstable among efficient assignments. Kwon and Shorrer (2019) introduce the notion of a blocking triplet that includes, in addition to a blocking pair, a student who violates the priority of the student in the blocking pair, and show that the EADA mechanism is minimally unstable among efficient assignments also when stability comparison is based on comparing (in the set-inclusion sense) sets of blocking triplets. Also, Kwon and Shorrer (2019) allow for private endowments, which is important to capture some other applications.

Ergin (2002) derived necessary and sufficient conditions on the priority profiles for the efficiency of the DA mechanism. Here, we ask a similar question and characterize the priority profiles for which there exists a Pareto improvement over the DA mechanism that is cardinally minimally unstable among efficient assignments. It turns out that whenever this is possible, such a mechanism must coincide with the EADA mechanism. ${ }^{10}$

In a recent paper, Bonkoungou and Nesterov (2020) use natural stability comparisons, including blocking-pair-wise and blocking-student-wise comparisons, to explain

\footnotetext{
${ }^{9}$ We say more about Abdulkadiroğlu et al. (2020) in Section 6.

${ }^{10}$ Doğan and Yenmez (2020), Dur et al. (2019), Ehlers and Morrill (2020), Kwon and Shorrer (2019), Tang and Zhang (2021), and Troyan et al. (2020) provide other justifications for EADA.
} 
some school choice reforms. In another recent paper, Combe et al. (2017) compare assignments in terms of their stability by comparing the sets of blocking pairs in the setinclusion sense, in the context of teacher assignments, where each teacher is initially endowed with a position at a school, and individual rationality and stability are incompatible.

\section{Model}

Let $N$ denote a finite set of students and let $C$ denote a finite set of schools. Each student $i \in N$ has a preference ordering $R_{i}$ over $C \cup\{\emptyset\},{ }^{11}$ where $\emptyset$ represents an outside option for the student. The strict part of the preference ordering $R_{i}$ is denoted by $P_{i}$, so if $c_{1}, c_{2} \in$ $C \cup\{\emptyset\}, c_{1} \neq c_{2}$, and $c_{1} R_{i} c_{2}$, then $c_{1} P_{i} c_{2}$. School $c$ is acceptable to student $i$ if the student prefers it to the outside option, that is, $c P_{i} \emptyset$. Each school $c \in C$ has a capacity $q_{c} \in \mathbb{N}$, which is the maximum number of students that the school can admit, and a priority ordering $\succeq_{c}$ over the set of students $N .{ }^{12}$ The strict part of the priority ordering $\succeq_{c}$ is denoted by $\succ_{c}$.

An assignment is a mapping $\mu: N \cup C \rightarrow N \cup C \cup\{\varnothing\}$ such that

(i) for each $i \in N, \mu(i) \in C \cup\{\emptyset\}$

(ii) for each $c \in C, \mu(c) \subseteq N$ such that $|\mu(c)| \leq q_{c}$

(iii) for each $i \in N$ and each $c \in C, i \in \mu(c)$ if and only if $c=\mu(i)$.

Let $\mathcal{A}$ denote the set of all assignments.

An assignment $\mu$ is individually rational if for each $i \in N, \mu(i) R_{i} \emptyset$. An assignment $\mu$ Pareto improves an assignment $\mu^{\prime}$ if for each $i \in N, \mu(i) R_{i} \mu^{\prime}(i)$ and there exists $j \in N$ such that $\mu(j) P_{j} \mu^{\prime}(j)$. An assignment $\mu$ is efficient if it cannot be Pareto improved.

A pair $(i, c) \in N \times C$ blocks $\mu$ if $c P_{i} \mu(i)$ and $\left[|\mu(c)|<q_{c}\right.$ or there exists $j \in \mu(c)$ such that $i \succ_{c} j$ ]. Let

$$
B(\mu)=\{(i, c) \in N \times C:(i, c) \text { blocks } \mu\}
$$

denote the set of blocking pairs at $\mu$ and let $B_{i}(\mu)=B(\mu) \cap(\{i\} \times C)$ denote the set of blocking pairs at $\mu$ containing student $i$.

An assignment $\mu$ is stable if it is individually rational and includes no blocking pair. Unfortunately, there exist school choice problems without an assignment that is both efficient and stable (Roth (1982)). We investigate assignments that are minimally unstable among efficient assignments based on methods to compare assignments by their stability.

A (school choice) problem $P$ is a tuple $(N, C, R, q, \succeq)$, where $R=\left(R_{i}\right)_{i \in N}$ denotes the (student) preference profile, $q=\left(q_{c}\right)_{c \in C}$ denotes the (school) capacity profile, and

\footnotetext{
${ }^{11}$ Formally, a preference ordering over $C \cup\{\emptyset\}$ is a complete, transitive, and antisymmetric binary relation over $C \cup\{\emptyset\}$. Binary relation $R_{i}$ over $C \cup\{\emptyset\}$ is complete if, for every $c_{1}, c_{2} \in C \cup\{\emptyset\}, c_{1} R_{i} c_{2}$ or $c_{2} R_{i} c_{1}$. It is transitive if, for every $c_{1}, c_{2}, c_{3} \in C \cup\{\emptyset\}, c_{1} R_{i} c_{2}$ and $c_{2} R_{i} c_{3}$ imply $c_{1} R_{i} c_{3}$. It is antisymmetric if, for every $c_{1}, c_{2} \in C \cup\{\emptyset\}, c_{1} R_{i} c_{2}$ and $c_{2} R_{i} c_{1}$ imply $c_{1}=c_{2}$.

${ }^{12}$ The priority ordering $\succeq_{c}$ is a complete, transitive, and antisymmetric binary relation over $N$.
} 
$\succeq=\left(\succeq_{c}\right)_{c \in C}$ denotes the (school) priority profile. We keep everything fixed except for the preference profile and, for short, a problem is often denoted by $R$. Let $\mathcal{P}$ denote the set of all problems.

\subsection{Stability comparisons}

A stability comparison is a function $f$ associating with each problem $P \in \mathcal{P}$ a binary relation over assignments $\gtrsim_{f}^{P} \subseteq \mathcal{A} \times \mathcal{A}$. We write $\mu \gtrsim_{f}^{P} \nu$ instead of $(\mu, \nu) \in \gtrsim_{f}^{P}$ and write $\mu \gtrsim_{f}^{P} \nu$ instead of $\left[\mu \gtrsim_{f}^{P} \nu\right.$ and not $\left.\nu \gtrsim_{f}^{P} \mu\right]$. We read $\mu \gtrsim_{f}^{E} \nu$ as " $\mu$ is weakly $f$-more stable than $\nu$ at $P$ " and $\mu \underset{\nsim f}{\gtrless} \nu$ as " $\mu$ is (strictly) $f$-more stable than $\nu$ at $P$ ". ${ }^{13}$ We say that $\mu$ is $f$-minimally unstable at $P$ among efficient assignments if $\mu$ is efficient and there exists no efficient assignment $\nu$ such that $\nu \underset{\nsim f}{\longrightarrow} \mu$. We use the abbreviation a.e.a. to denote "among efficient assignments." Below, we describe two primitive stability comparisons based on blocking pairs.

The blocking pairs inclusion comparison (pincl) is defined as follows. For each problem $P \in \mathcal{P}$ and $\mu, \nu \in \mathcal{A}$,

$$
\mu \gtrsim_{\text {pincl }}^{P} \nu \Leftrightarrow B(\mu) \subseteq B(\nu) .
$$

The blocking pairs cardinality comparison (pcard) is defined as follows. For each problem $P \in \mathcal{P}$ and $\mu, \nu \in \mathcal{A}$,

$$
\mu \gtrsim_{\text {pcard }}^{P} \nu \Leftrightarrow|B(\mu)| \leq|B(\nu)| .
$$

Note that any two assignments can be compared with respect to blocking pairs cardinality, but not necessarily with respect to blocking pairs inclusion. We use the convention to write more stable instead of pincl more stable, minimally unstable instead of pincl minimally unstable, cardinally more stable instead of pcard more stable, and cardinally minimally unstable instead of pcard minimally unstable. ${ }^{14}$

REMARK 1. For any stable assignment $\mu$, there is no other assignment that is (cardinally) more stable than $\mu$. However, $\mu$ is not necessarily minimally unstable among efficient assignments, since $\mu$ may not be efficient.

\subsection{Mechanisms}

A mechanism associates each problem with an assignment. When we say that a mechanism satisfies a certain assignment property (such as efficiency or minimal instability among efficient assignments), we mean that at each problem, the assignment prescribed by the mechanism satisfies the property.

\footnotetext{
${ }^{13}$ Let $\mathcal{L}$ denote the set of all binary relations over assignments. Given $\gtrsim \in \mathcal{L}$, (i) $\gtrsim$ is complete if for all $\mu, \nu \in \mathcal{A}$, we have $\mu \gtrsim \nu$ or $\nu \gtrsim \mu$ and (ii) $\gtrsim$ is transitive if $\mu \gtrsim \nu$ and $\nu \gtrsim \eta$ imply $\mu \gtrsim \eta$.

${ }^{14}$ Note that $\gtrsim_{\text {pincl }}^{P}$ is transitive but not complete, and $\gtrsim_{\text {pcard }}^{P}$ is complete (as any two assignments can be compared) and transitive. Moreover, $\gtrsim_{\text {pincl }}^{P} \subseteq \gtrsim_{\text {pcard }}^{P}$.
} 
The deferred acceptance (DA) mechanism due to Gale and Shapley (1962) is used in many school districts that have reformed their school choice systems. The DA mechanism associates each problem $P$ with the assignment determined by the following deferred acceptance algorithm.

Deferred Acceptance Algorithm. Step 1. Each student proposes to her top-ranked acceptable school. If there is no such school, then she is assigned to her outside option. Each school $c$ considers the set of proposals that it receives. Among them, it tentatively accepts the highest priority students up to its capacity and rejects the others. If there is no rejection, then stop.

Step $t \geq 2$. Each student who is rejected at Step $t-1$ proposes to her top-ranked acceptable school among those that have not rejected her yet. If there is no such school, then she is assigned to her outside option. Each school $c$ considers the set of students that it tentatively accepted at Step $t-1$ together with students that have proposed at Step $t$. Among them, it tentatively accepts the highest priority students up to its capacity and rejects the others. If there is no rejection, then stop. Otherwise, move to Step $t+1$.

The DA algorithm stops in finitely many steps and the DA assignment, which we denote by $\mathrm{DA}(P)$, is defined by the acceptances at the last step. At each problem, the DA assignment is stable, but not necessarily efficient (Abdulkadiroğlu and Sönmez (2003)).

Another mechanism, which was proposed as an efficient mechanism that Pareto improves over the DA mechanism, turns out to be central to our analysis. The efficiencyadjusted deferred acceptance mechanism (Kesten (2010)) is based on the EADA algorithm, which works by iteratively removing certain schools from the preference orderings of certain students and rerunning the DA algorithm. Instead of providing Kesten's original definition of the EADA algorithm, we provide the outcome-equivalent version due to Tang and Yu (2014). Given an assignment $\mu$, a school $c \in C$ is underdemanded at $\mu$ if no student strictly prefers it to his assigned school. We adopt the convention that for each student $i \in N$, his outside option is underdemanded at any assignment.

Efficiency-Adjusted Deferred Acceptance Algorithm. Round 0. Run DA for the problem $P=(N, C, R, q, \succeq)$.

Round $r \geq 1$. Identify underdemanded schools at the outcome of round $r-1$. Let $I_{r}$ denote the set of students who are assigned to underdemanded schools (including the students who are assigned to their outside options). Let $\mu^{r}$ denote the restriction of the outcome of Round $r-1$ to $I_{r}$. (Note that $\mu^{r}$ includes only the underdemanded schools and students in $I_{r}$.) Remove these schools and $I_{r}$ from the problem. Stop if there are no remaining schools. Otherwise, run DA for the reduced problem. Move to the next round, Round $r+1$.

The EADA algorithm stops in finitely many rounds, say in $m$ rounds. The EADA assignment is defined as the collection of $\mu^{1}, \ldots, \mu^{m}$; that is, $\operatorname{EADA}(P)=\mu^{1} \cup \cdots \cup \mu^{m}$. For 
each $t \in\{1, \ldots, m\}$, let $\mu_{t}$ denote the assignment obtained by collecting $\mu^{1}, \ldots, \mu^{t}$ together with the DA assignment for the reduced problem at the end of Round $t$. In other words, $\mu_{t}$ is the assignment obtained by iterating the EADA algorithm for only $t$ rounds. Note that $\mu_{m}=\operatorname{EADA}(P)$.

At each problem, the EADA assignment is efficient and Pareto improves the DA assignment (Tang and Yu (2014), Kesten (2010)).

Remark 2. Given a problem, if the EADA assignment coincides with the DA assignment, then the EADA assignment is the unique assignment that is (cardinally) minimally unstable among efficient assignments.

\section{The MAIN RESULT}

It turns out that there exists a Pareto improvement over the DA mechanism that is minimally unstable among efficient assignments: the efficiency adjusted deferred acceptance (EADA) mechanism (Kesten (2010)). ${ }^{15}$ Noting that minimal instability is a relatively weak requirement, as many assignments are incomparable when their sets of blocking pairs are compared, we turn our focus to cardinal minimal instability, which results in the following impossibilities.

(a) There is no Pareto improvement over the DA mechanism that is cardinally minimally unstable among efficient assignments.

(b) Any Pareto improvement over the DA mechanism may produce an arbitrarily large loss in terms of cardinal minimal instability, that is, there exists a problem with an alternative efficient assignment that has arbitrarily fewer blocking pairs.

The proofs of these results and a general analysis of impossibilities with minimally unstable Pareto improvements over DA is presented in Section 5. In this section, motivated by these impossibilities, we characterize the priority profiles for which there exists a Pareto improvement over the DA assignment that is cardinally minimally unstable among efficient assignments at each problem. ${ }^{16}$

We investigate this question under the following two assumptions:

A1: Unit capacities. Each school has unit capacity, i.e., $q_{c}=1$ for each $c \in C$.

A2: At least five schools. There are at least five schools, i.e., $|C| \geq 5$.

The following notions are useful. A priority profile $\left(\succeq_{c}\right)_{c \in C}$ includes an Ergin cycle (Ergin (2002)) if there exist a list of three students $\left(i_{1}, i_{2}, i_{3}\right)$ and a pair of schools $\left(c_{1}, c_{2}\right)$ such that $i_{3} \succ_{c_{1}} i_{1} \succ_{c_{1}} i_{2}$ and $i_{2} \succ_{c_{2}} i_{3}$. We call student $i_{1}$ the initiator of the cycle.

\footnotetext{
${ }^{15}$ This result also follows from Tang and Zhang (2021). We thank Szilvia Papai for bringing this to our attention. We present our proof, which is independent and different from Tang and Zhang (2021), in Appendix A.1.

${ }^{16}$ This is parallel to Ergin (2002), who derived necessary and sufficient conditions (on the capacitypriority profile) for the efficiency of the DA mechanism. Recall that the DA mechanism is stable but not necessarily efficient.
} 
We say that a list of three students $\left(i_{1}, i_{2}, i_{3}\right)$ and a pair of schools $\left(c_{1}, c_{2}\right)$ constitute a tight Ergin cycle if they constitute an Ergin cycle and both

I. there is no $m \in N \backslash\left\{i_{2}, i_{3}\right\}$ such that $i_{2} \succ_{c_{2}} m \succ_{c_{2}} i_{3}$

II. there is no $m \in N \backslash\left\{i_{1}, i_{2}, i_{3}\right\}$ such that $i_{3} \succ_{c_{1}} m \succ_{c_{1}} i_{2}$.

Given two Ergin cycles consisting of $\left(i_{1}, i_{2}, i_{3}\right),\left(c_{1}, c_{2}\right)$ and $\left(j_{1}, j_{2}, j_{3}\right),\left(c_{1}^{\prime}, c_{2}^{\prime}\right)$, respectively, we say that the Ergin cycles are distinct if all the students and schools in the two Ergin cycles are distinct, i.e., $\left\{i_{1}, i_{2}, i_{3}\right\} \cap\left\{j_{1}, j_{2}, j_{3}\right\}=\emptyset$ and $\left\{c_{1}, c_{2}\right\} \cap\left\{c_{1}^{\prime}, c_{2}^{\prime}\right\}=\emptyset$. We say that the two Ergin cycles are distinct except for the initiator if $i_{1}=j_{1}$ and all the other students and schools in the two Ergin cycles are distinct.

We introduce the following three conditions on a priority profile $\left(\succeq_{c}\right)_{c \in C}$.

Condition 1. All Ergin cycles are tight.

Condition 2. There are no two Ergin cycles that are distinct except for the initiator.

Condition 3. There are no two distinct Ergin cycles.

Our main result is that these three conditions are necessary and sufficient for the existence of a Pareto improvement over the DA mechanism that is cardinally minimally unstable among efficient assignments at each problem. ${ }^{17}$ Moreoever, whenever such a Pareto improvement exists, it coincides with the EADA mechanism.

THEOREM 1. Suppose each school has unit capacity and there are at least five schools. The following statements are equivalent:

(i) There exists a mechanism that is both a Pareto improvement over the DA mechanism and cardinally minimally unstable among efficient assignments.

(ii) The priority profile $\succeq$ satisfies Conditions 1, 2, and 3 .

(iii) There is a unique mechanism that is both a Pareto improvement over the DA mechanism and cardinally minimally unstable among efficient assignments (and, therefore, this mechanism coincides with the EADA mechanism).

Before providing a complete proof, we provide rough intuitions for why these three conditions are necessary. First note that when the DA assignment is not efficient, a simple Pareto improvement over the DA assignment involves reassignment of the assigned seats of students in an Ergin cycle while keeping the assignment of the initiator of the cycle fixed. This improvement creates at least one blocking pair (since the initiator is now involved in a blocking pair), and the exact number of resulting blocking pairs essentially depends on the relative position of the initiator in the priority orderings of all the schools

\footnotetext{
${ }^{17}$ The counterparts of the characterizing conditions are still necessary for the EADA mechanism to be cardinally minimally unstable among efficient assignments in the general multicapacity case (Appendix A.2), and, therefore, the main insights extend to the general setup.
} 
in the Ergin cycle. (Note that only the position of the initiator in the first school's priority ordering is relevant in the definition of an Ergin cycle.) When Condition 1 is violated, it is possible to construct a preference profile such that the initiator is included in blocking pairs with several schools in the unique Pareto improvement over the DA assignment, while there is an alternative efficient assignment with smaller number of blocking pairs where the initiator is assigned his most preferred school in the cycle and is not included in any blocking pair. When Condition 2 is violated, it is possible to construct a preference profile such that the same student initiates two distinct rejection cycles consecutively such that the initiator is included in blocking pairs with several schools from both cycles in the unique Pareto improvement over the DA assignment, while there is an alternative efficient assignment with a smaller number of blocking pairs (although not a Pareto improvement over DA) where the initiator is assigned his most preferred school in the two cycles and is not included in any blocking pair. When Condition 3 is violated, it is possible to construct a preference profile such that a student initiates a rejection cycle, then a different student-as a result of the first rejection cycle-initiates a different rejection cycle such that both initiators are included in blocking pairs with schools from their cycles in the unique Pareto improvement over the DA assignment, while there is an alternative efficient assignment with smaller number of blocking pairs where both initiators are assigned their most preferred schools in the two cycles and are not included in any blocking pair.

Note that the existence of a mechanism that is cardinally minimally unstable a.e.a. is trivial. The surprising insight of Theorem 1 is that there is only one that is a Pareto improvement over the DA mechanism, namely the EADA mechanism. The DA mechanism has been used in many different contexts, and economists have proposed efficient Pareto improvements of DA. Now if cardinal minimal instability is important, then Theorem 1 describes the priority structures for which this can be done (à la Ergin (2002) for the compatibility of efficiency and stability).

REMARK 3. Instead of simply counting blocking pairs, one might consider other cardinal stability comparisons that do not treat all blocking pairs the same way. One natural method is to distinguish blocking pairs based on the number of positions the student and the school improve. Formally, for problem $P$ and assignment $\mu$, the blocking pair $(i, c)$ is an $(+k,+l)$ instance if the rank of $c$ in $i$ 's preference is $k$ positions above $\mu(i)$ and $i$ is $l$ positions above $\mu(c)$. For short, we write $\perp(i, c)=(+k,+l)$ and $\perp(B(\mu))=\{\perp(i, c):(i, c) \in B(\mu)\}$ (where repetitions of the same instance are allowed). Let us call $f$ cardinal if for any problem $P$ and all $\mu$ and $\nu, \perp(B(\mu))=\perp(B(\nu))$ implies $\mu \sim_{f}^{P} \nu$. Let us call $f$ reasonable if for any problem $P$ and all $\mu$ and $\nu$ such that $\perp(B(\mu))=\{(+1,+1)\}$ and $|B(\nu)|>1$, we have $\mu \underset{\sim f}{\gtrless} \nu$. Now Theorem 1 remains unchanged when the blocking pairs cardinality comparison is replaced by any reasonable cardinal stability comparison $f .^{18}$

\footnotetext{
${ }^{18}$ This is due to the fact that in the proof of (ii) $\Rightarrow$ (iii) of Theorem 1, all the examples have $\perp\left(B\left(\mu^{\prime}\right)\right)=$ $\{(+1,+1)\}$ and $|B(\mu)|>1$, and, hence, $\mu^{\prime} \underset{{ }_{f}}{P_{P}^{P}} \mu$.
} 
REMARK 4. (a) The proof of Theorem 1 shows that if the priority profile satisfies Conditions 1,2, and 3, then for each problem, either the DA assignment is efficient or there exists a unique Pareto improvement of the DA assignment that contains exactly one blocking pair. Therefore, the latter assignment coincides with the EADA assignment. Thus, the EADA assignment is as close to stability as possible if it is unstable (by allowing only one blocking pair). We call an assignment (almost) stable if it is stable or contains exactly one blocking pair. Now Theorem 1 shows that for the EADA mechanism, cardinal minimal instability a.e.a. implies (almost) stability.

(b) As the set of efficient Pareto improvements over DA is a subset of the efficient assignments, Conditions 1, 2, and 3 are sufficient for the EADA mechanism to be cardinally minimally unstable among efficient Pareto improvements over DA. It is an open question to determine necessary and sufficient conditions for the EADA mechanism to be cardinally minimally unstable among efficient Pareto improvements over DA.

\subsection{Proof of Theorem 1}

Note that (iii) $\Rightarrow$ (i) is trivial. We prove (i) $\Rightarrow$ (ii) and (ii) $\Rightarrow$ (iii).

(i) $\Rightarrow$ (ii). We show that if one of Conditions 1,2 , or 3 is violated, then there exist problems where no efficient Pareto improvement over DA is cardinally minimally unstable.

Lemma 1. Suppose that the priority profile violates Condition 1, i.e., it includes an Ergin cycle that is not tight. Then there exists a problem such that there is no Pareto improvement over DA that is cardinally minimally unstable among efficient assignments.

Proof. Suppose that a list of students $(1,2,3)$ and a list of schools $\left(c_{1}, c_{2}\right)$ constitute an Ergin cycle of $\left(\succeq_{c}\right)_{c \in C}$ that is not tight.

Case 1. Suppose that $2 \succ_{c_{2}} 1 \succ_{c_{2}} 3$. We depict only the relative positions of the students $\{1,2,3\}$ in $\succeq$ :

\begin{tabular}{cc}
$\succeq_{c_{1}} \succeq_{c_{2}}$ \\
\hline 3 & 2 \\
1 & 1 \\
2 & 3
\end{tabular}

Let $R$ be a preference profile such that the preference orderings of students in $\{1,2,3\}$ over their acceptable schools are

$$
\begin{array}{l|l|l}
R_{1} & R_{2} & R_{3} \\
\hline c_{1} & c_{1} & c_{2} \\
\hline c_{2} & c_{2} & c_{1} \\
\emptyset & \emptyset & \emptyset
\end{array}
$$

and each other student finds no school acceptable. 
Let $\mu$ be the assignment where the assignments of the students in $\{1,2,3\}$ are as depicted above in boxes, and each other student is assigned to his outside option.

Let $\mu^{\prime}$ be the assignment where the assignments of the students in $\{1,2,3\}$ are as underlined above, and each other student is assigned to his outside option.

Note that the DA assignment is $\left(\begin{array}{lll}1 & 2 & 3 \\ \varnothing & c_{2} & c_{1}\end{array}\right)$ (each other student is assigned to his outside option), and $\mu$ is the unique Pareto improvement over the DA assignment that is efficient and $\mu^{\prime}$ is an efficient assignment. Moreover, $B(\mu)=\left\{\left(1, c_{1}\right),\left(1, c_{2}\right)\right\}$ and $B\left(\mu^{\prime}\right)=\left\{\left(3, c_{1}\right)\right\}$.

Case 2. Suppose that there is $m \in N \backslash\{1,2,3\}$ such that $3 \succ_{c_{2}} m \succ_{c_{2}} 2$. We depict only the relative positions of the students $\{1,2,3, m\}$ in $\succeq$ :

\begin{tabular}{cc}
$\succeq_{c_{1}} \succeq_{c_{2}}$ \\
\hline 3 & 2 \\
1 & $m$ \\
2 & 3
\end{tabular}

Let $R$ be a preference profile such that the preference orderings of $\{1,2,3, m\}$ over their acceptable schools are

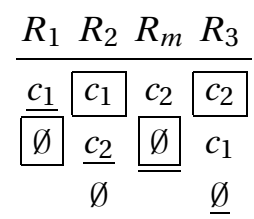

and each other student finds no school acceptable.

Note that in profile $R$, student 1 ranks $c_{2}$ unacceptable, and, thus, the DA assignment and any efficient assignment does not assign 1 to $c_{2}$ independently of the position of 1 in $\succeq_{c_{2}}$. Thus, the position of 1 in $\succeq_{c_{2}}$ is omitted. The same applies to student $m$ and $c_{1}$ (and similarly below, for any example where a student does not rank a certain school, we omit the student's position in the priority ordering of this school).

Let $\mu$ be the assignment where the assignments of the students in $\{1,2,3, m\}$ are as depicted above in boxes, and each other student is assigned to his outside option.

Let $\mu^{\prime}$ be the assignment where the assignments of the students in $\{1,2,3, m\}$ are as underlined above, and each other student is assigned to his outside option.

Note that the DA assignment is $\left(\begin{array}{llll}1 & 2 & m & 3 \\ \emptyset & c_{2} & \emptyset & c_{1}\end{array}\right)$ (each other student is assigned to his outside option), and $\mu$ is the unique Pareto improvement over the DA assignment that is efficient and $\mu^{\prime}$ is an efficient assignment. Moreover, $B(\mu)=\left\{\left(1, c_{1}\right),\left(m, c_{2}\right)\right\}$ and $B\left(\mu^{\prime}\right)=\left\{\left(3, c_{1}\right)\right\}$.

Case 3. Suppose that there is $m \in N \backslash\{1,2,3\}$ such that $3 \succ_{c_{1}} m \succ_{c_{1}} 2$. Without loss of generality, suppose that $m \succ_{c_{1}} 1$ (if $1 \succ_{c_{1}} m$, then we exchange the roles of $m$ and $c_{1}$ ). We depict only the relative positions of the students $\{1,2,3, m\}$ in $\succeq$ :

\begin{tabular}{cc}
$\succeq_{c_{1}} \succeq_{c_{2}}$ \\
\hline $3 \quad 2$ \\
$m \quad 3$ \\
1 \\
2
\end{tabular}


Let $R$ be a preference profile such that the preference orderings of $\{1,2,3, m\}$ over their acceptable schools are

$$
\begin{array}{lllll}
R_{1} & R_{2} & R_{3} & R_{m} \\
\hline c_{1} & c_{1} & c_{2} & \frac{c_{1}}{} \\
\emptyset & \frac{c_{2}}{c_{1}} & c_{1} & \emptyset \\
\hline & \varnothing & \emptyset &
\end{array}
$$

and each other student finds no school acceptable.

Let $\mu$ be the assignment where the assignments of the students in $\{1,2,3, m\}$ are as depicted above in boxes, and each other student is assigned to his outside option.

Let $\mu^{\prime}$ be the assignment where the assignments of the students in $\{1,2,3, m\}$ are as underlined above, and each other student is assigned to his outside option.

Note that the DA assignment is $\left(\begin{array}{llll}1 & 2 & 3 & m \\ \emptyset & c_{2} & c_{1} & \emptyset\end{array}\right)$ (each other student is assigned to his outside option), and $\mu$ is the unique Pareto improvement over the DA assignment that is efficient and $\mu^{\prime}$ is an efficient assignment. Moreover, $B(\mu)=\left\{\left(1, c_{1}\right),\left(m, c_{1}\right)\right\}$ and $B\left(\mu^{\prime}\right)=\left\{\left(3, c_{1}\right)\right\}$.

Lemma 2. Suppose that the priority profile violates Condition 2, i.e., it includes two Ergin cycles that are distinct except for the initiator. Then there exists a problem such that there is no Pareto improvement over DA that is cardinally minimally unstable among efficient assignments.

Proof. Suppose that there are two Ergin cycles of $\left(\succeq_{c}\right)_{c \in C}$ consisting of $\left(i_{1}, i_{2}, i_{3}\right)$, $\left(c_{1}, c_{2}\right)$ and $\left(j_{1}, j_{2}, j_{3}\right),\left(c_{1}^{\prime}, c_{2}^{\prime}\right)$, that are distinct except for the initiator. Let $i_{1}=j_{1} \equiv i$ :

\begin{tabular}{cccc}
$\succeq_{c_{1}} \succeq_{c_{2}} \succeq_{c_{1}^{\prime}} \succeq_{c_{2}^{\prime}}$ \\
\hline$i_{3}$ & $i_{2}$ & $j_{3}$ & $j_{2}$ \\
$i$ & $i_{3}$ & $i$ & $j_{3}$ \\
$i_{2}$ & & $j_{2}$ &
\end{tabular}

Let $R$ be a preference profile such that the preference orderings of students in $\left\{i, i_{2}, i_{3}, j_{2}, j_{3}\right\}$ over their acceptable schools are

$$
\begin{array}{ccccc}
R_{i} & R_{i_{2}} & R_{i_{3}} & R_{j_{2}} & R_{j_{3}} \\
\hline c_{1} & c_{1} & c_{2} & c_{1}^{\prime} & c_{2}^{\prime} \\
\hline c_{1}^{\prime} & c_{2} & c_{1} & \overline{c_{2}^{\prime}} & \underline{c_{1}^{\prime}} \\
\emptyset & \emptyset & \emptyset & \emptyset & \emptyset
\end{array}
$$

and each other student finds no school acceptable.

Let $\mu$ be the assignment where the assignments of the students in $\left\{i, i_{2}, i_{3}, j_{2}, j_{3}\right\}$ are as depicted above in boxes, and each other student is assigned to his outside option.

Let $\mu^{\prime}$ be the assignment where the assignments of the students in $\left\{i, i_{2}, i_{3}, j_{2}, j_{3}\right\}$ are as underlined above, and each other student is assigned to his outside option.

Note that the DA assignment is $\left(\begin{array}{lllll}i & c_{2} & i_{3} & j_{2} & j_{3} \\ \emptyset & c_{2} & c_{1} & c_{2}^{\prime} & c_{1}^{\prime}\end{array}\right)$ (each other student is assigned to his outside option), and $\mu$ is the unique Pareto improvement over DA that is efficient and $\mu^{\prime}$ is an efficient assignment. Moreover, $B(\mu)=\left\{\left(i, c_{1}\right),\left(i, c_{1}^{\prime}\right)\right\}$ and $B\left(\mu^{\prime}\right)=\left\{\left(i_{3}, c_{1}\right)\right\}$. 
Lemma 3. Suppose that the priority profile violates Condition 3, i.e., it includes two distinct Ergin cycles. Then there exists a problem such that there is no Pareto improvement over DA that is cardinally minimally unstable among efficient assignments.

Proof. Suppose that there are two distinct generalized cycles of $\left(\succeq_{c}\right)_{c \in C}$ consisting of $\left(i_{1}, i_{2}, i_{3}\right),\left(c_{1}, c_{2}\right)$ and $\left(j_{1}, j_{2}, j_{3}\right),\left(c_{1}^{\prime}, c_{2}^{\prime}\right)$. We depict only the relevant relative positions of the students $\left(i_{1}, i_{2}, i_{3}, j_{1}, j_{2}, j_{3}\right)$ in $\succeq$ :

\begin{tabular}{cccc}
$\succeq_{c_{1}} \succeq_{c_{2}} \succeq_{c_{1}^{\prime}} \succeq_{c_{2}^{\prime}}$ \\
\hline$i_{3}$ & $i_{2}$ & $j_{3}$ & $j_{2}$ \\
$i_{1}$ & $i_{3}$ & $j_{1}$ & $j_{3}$ \\
$i_{2}$ & & $j_{2}$ &
\end{tabular}

Since $|C| \geq 5$, there exists $c \in C \backslash\left\{c_{1}, c_{2}, c_{1}^{\prime}, c_{2}^{\prime}\right\}$. Without loss of generality, suppose that $j_{1} \succ_{c} i_{1}$.

Let $R$ be a preference profile such that the preference orderings of students in $\left(i_{1}, i_{2}, i_{3}, j_{1}, j_{2}, j_{3}\right)$ over their acceptable schools are

\begin{tabular}{|c|c|c|c|c|}
\hline \multicolumn{5}{|c|}{$R_{i_{1}} R_{i_{2}} R_{i_{3}} R_{j_{1}} R_{j_{2}} R_{j_{3}}$} \\
\hline$\underline{c}$ & $c_{1}$ & $c_{2} c_{1}^{\prime}$ & $c_{1}^{\prime}$ & \\
\hline$c_{1}$ & $c_{2}$ & $\begin{array}{c}c_{1} \quad c \\
\quad\end{array}$ & $c_{2}^{\prime}$ & \\
\hline$\varnothing$ & $\varnothing$ & $\varnothing \varnothing$ & $\underline{\emptyset}$ & \\
\hline
\end{tabular}

and each other student finds no school acceptable.

Let $\mu$ be the assignment where the assignments of the students in $\left(i_{1}, i_{2}, i_{3}, j_{1}, j_{2}, j_{3}\right)$ are as depicted above in boxes, and each other student is assigned to his outside option.

Let $\mu^{\prime}$ be the assignment where the assignments of the students in $\left(i_{1}, i_{2}, i_{3}, j_{1}, j_{2}, j_{3}\right)$ are as underlined above, and each other student is assigned to his outside option.

Note that the DA assignment is $\left(\begin{array}{llllll}i_{1} & i_{2} & i_{3} & j_{1} & j_{2} & j_{3} \\ \emptyset & c_{2} & c_{1} & c & c_{2}^{\prime} & c_{1}^{\prime}\end{array}\right)$ (each other student is assigned to his outside option), and $\mu$ is the unique Pareto improvement over DA that is efficient and $\mu^{\prime}$ is an efficient assignment. Moreover, $B(\mu)=\left\{\left(i_{1}, c_{1}\right),\left(j_{1}, c_{1}^{\prime}\right)\right\}$ and $B\left(\mu^{\prime}\right)=\left\{\left(j_{2}, c_{2}^{\prime}\right)\right\}$.

(ii) $\Rightarrow$ (iii). We now show that if Conditions 1, 2, and 3 are satisfied, then the EADA mechanism is the unique Pareto improvement over the DA mechanism that is cardinally minimally unstable among efficient assignments. First, we define some auxiliary notions and prove some auxiliary results.

A priority profile $\left(\succeq_{c}\right)_{c \in C}$ includes a generalized cycle (of length $n-1$ ) if there exist a list of students $(1, \ldots, n)$ and a list of schools $\left(c_{1}, \ldots, c_{n-1}\right)$ such that $n \succ_{c_{1}} 1 \succ_{c_{1}} 2$ and for each $i \in\{2, \ldots, n-1\}, i \succ_{c_{i}} i+1 .{ }^{19}$ Given a generalized cycle consisting of $(1, \ldots, n)$ and $\left(c_{1}, \ldots, c_{n-1}\right)$, we call the first student, student 1 , the initiator of the generalized cycle. ${ }^{20}$

Given two generalized cycles consisting of $\left(i_{1}, \ldots, i_{n}\right),\left(c_{1}, \ldots, c_{n-1}\right)$ and $\left(j_{1}, \ldots, j_{m}\right)$, $\left(c_{1}^{\prime}, \ldots, c_{m-1}^{\prime}\right)$, respectively, we say that the two generalized cycles are distinct if all

${ }^{19}$ The notion of a generalized cycle was first introduced in Ergin (2002).

${ }^{20}$ Note that in the definition of a generalized cycle, the first school $c_{1}$ and the first student 1 have particular roles; in that sense, rotating the elements of a generalized cycle would not necessarily result in a new generalized cycle, in contrast to what the word "cycle" would normally indicate. 
the students and schools in the two generalized cycles are distinct, i.e., $\left\{i_{1}, \ldots, i_{n}\right\} \cap$ $\left\{j_{1}, \ldots, j_{m}\right\}=\emptyset$ and $\left\{c_{1}, \ldots, c_{n-1}\right\} \cap\left\{c_{1}^{\prime}, \ldots, c_{m-1}^{\prime}\right\}=\emptyset$. We say that the two generalized cycles are distinct except for the initiator if $i_{1}=j_{1}$ and all the other students and schools in the two generalized cycles are distinct.

We say that a list of students $(1, \ldots, n)$ and a list of schools $\left(c_{1}, \ldots, c_{n-1}\right)$ constitute a tight generalized cycle (of length $n-1$ ) if they constitute a generalized cycle and

I. there is no $m \in N \backslash\{2, \ldots, n\}$ and $k \in\{2, \ldots, n-1\}$ such that $k \succ_{c_{k}} m \succ_{c_{k}} k+1$

II. there is no $m \in N \backslash\{1,2, \ldots, n\}$ such that $n \succ_{c_{1}} m \succ_{c_{1}} 2$.

Lемма 4. If the priority profile satisfies Condition 3, then there are no two distinct generalized cycles.

Proof. Note that Ergin cycles are generalized cycles of length 2. Thus, if there are two distinct Ergin cyles, then there are two distinct generalized cycles. Furthermore, if there are two distinct generalized cycles, then by Ergin (2002) (Step 2 in the proof of Theorem 1), there are two distinct Ergin cycles.

Lemma 5. If the priority profile satisfies Conditions 2 and 3 , then there are no two generalized cycles that are distinct except for the initiator.

Proof. Consider any two generalized cycles consisting of $\left(i_{1}, \ldots, i_{n}\right),\left(c_{1}, \ldots, c_{n-1}\right)$ and $\left(j_{1}, \ldots, j_{m}\right),\left(c_{1}^{\prime}, \ldots, c_{m-1}^{\prime}\right)$ that are distinct except for the initiator. Then $i_{1}=j_{1}$ and all the other students and schools in the two generalized cycles are distinct. But then, by Ergin (2002) (Step 2 in the proof of Theorem 1), either there exist two distinct Ergin cycles, which implies that Condition 3 is violated, or there exist two Ergin cycles that are distinct except for the initiator, which implies that Condition 2 is violated.

Lемма 6. If the priority profile satisfies Conditions 1,2, and 3, then all generalized cycles are tight.

Proof. Suppose that there is a generalized cycle that is not tight. Then there are a list of students $(1, \ldots, n)$ and a list of schools $\left(c_{1}, \ldots, c_{n-1}\right)$ such that one of the following statements holds:

(i) There is $m \in N \backslash\{1,2, \ldots, n\}$ such that $n \succ_{c_{1}} m \succ_{c_{1}} 2$.

(ii) There is $m \in N \backslash\{2, \ldots, n\}$ and $k \in\{2, \ldots, n-1\}$ such that $k \succ_{c_{k}} m \succ_{c_{k}} k+1$.

If $n-1=2$, then there is an Ergin cycle that is not tight, which implies that Condition 1 is violated. So suppose that $n-1>2$.

Step 1. Suppose that (i) is satisfied. Without loss of generality, suppose that there is no shorter generalized cycle that satisfies (i). If $2 \succ_{c_{2}} n$, then there exists an Ergin cycle that is not tight, consisting of $(1,2, n)$ and $\left(c_{1}, c_{2}\right)$, which violates Conditiion 1 . Similarly, if there exists $l \in\{1, \ldots, n-1\}$ such that $2 \succ_{c_{l}} n$, then there exists an Ergin cycle that is not tight. So suppose that $n \succ_{c_{l}} 2$ for all $l \in\{1, \ldots, n-1\}$. 
Now, if $n-2 \succ_{c_{n-1}} n-1 \succ_{c_{n-1}} n \succ_{c_{n-1}} 2$, then there is a shorter generalized cycle that satisfies (i), consisting of $(n, 2,3, \ldots, n-3, n-2)$ and $\left(c_{n-1}, c_{2}, \ldots, c_{n-2}\right)$, a contradiction. If $n-1 \succ_{c_{n-1}} n-2 \succ_{c_{n-1}} n \succ_{c_{n-1}} 2$, then, again, there is a shorter generalized cycle that satisfies (i), consisting of $(1,2, \ldots, n-2, n)$ and $\left(c_{1}, \ldots, c_{n-3}, c_{n-1}\right)$, a contradiction. If $n-1 \succ_{c_{n-1}} n \succ_{c_{n-1}} n-2 \succ_{c_{n-1}} 2$, then, again, there is a shorter generalized cycle that satisfies (i), consisting of $(n-2,2,3, \ldots, n-2, n-1)$ and $\left(c_{n-1}, c_{2}, c_{3}, \ldots, c_{n-2}, c_{n-1}\right)$, a contradiction. If $n-1 \succ_{c_{n-1}} n \succ_{c_{n-1}} 2 \succ_{c_{n-1}} n-2$, then there exists an Ergin cycle that is not tight, consisting of $(2, n-2, n-1)$ and $\left(c_{n-1}, c_{n-2}\right)$, which is a violation of Condition 1 , a contradiction. Hence (i) cannot be satisfied.

Step 2. Suppose that (i) is not satisfied, but (ii) is satisfied. Without loss of generality, suppose that there is no shorter generalized cycle that satisfies (ii).

Suppose that $m \neq 1$. If $k \succ_{c_{k}} 1 \succ_{c_{k}} k+1$, clearly there is a generalized cycle that satisfies (i), a contradiction. Thus, $1 \succ_{c_{k}} k$ or $k+1 \succ_{c_{k}}$ 1. If $1 \succ_{c_{k}} k$, then the generalized cycle consisting of $(k, k+1, \ldots, n, 1)$ and $\left(c_{k}, \ldots, c_{n-1}, c_{1}\right)$ satisfies (i) by $1 \succ_{c_{k}} m \succ_{c_{k}} k+1$, a contradiction. If $k+1 \succ_{c_{k}} 1$, then the generalized cycle consisting of $(k+1,1,2, \ldots, k)$ and $\left(c_{k}, c_{1}, \ldots, c_{k-1}\right)$ satisfies (i) by $k \succ_{c_{k}} m \succ_{c_{k}} 1$, a contradiction.

Suppose that $m=1$. If $k+1 \succ_{c_{k}} n$, then the generalized cycle consisting of ( $k+$ $1, n, 2,3, \ldots, k)$ and $\left(c_{k}, c_{1}, c_{2}, \ldots, c_{k-1}\right)$ satisfies (i) by $k \succ_{c_{k}} 1 \succ_{c_{k}} k+1$, a contradiction. Similarly, if $n \succ_{c_{k}} k$, then the generalized cycle consisting of $(k, k+1, \ldots, n)$ and $\left(c_{k}, c_{k+1}, \ldots, c_{n}\right)$ satisfies (i) by $k \succ_{c_{k}} 1 \succ_{c_{k}} k+1$, a contradiction. Thus, $k \succ_{c_{k}} n \succ_{c_{k}}$ $k+1$. If $k+1 \succ_{c_{1}} 2$, then the generalized cycle consisting of $(n, k+1,2, \ldots, k)$ and $\left(c_{k}, c_{1}, \ldots, c_{k-1}\right)$ satisfies (i) by $k \succ_{c_{k}} 1 \succ_{c_{k}} k+1$, a contradiction. Thus, $2 \succ_{c_{1}} k+1$. But then the generalized cycle consisting of $(2, k+1, k+2, \ldots, n)$ and $\left(c_{1}, c_{k+1}, \ldots, c_{n-1}\right)$ satisfies (i) by $n \succ_{c_{1}} 1 \succ_{c_{1}} k+1$, a contradiction.

Given an assignment $\mu$, a list of students $\left(i_{1}, \ldots, i_{k}\right)$ is called an improvement cy$c l e^{21}$ if $\mu\left(i_{t+1}\right) P_{i_{t}} \mu\left(i_{t}\right)$ for each $t \in\{1, \ldots, k-1\}$ and $\mu\left(i_{1}\right) P_{i_{k}} \mu\left(i_{k}\right){ }^{22}$ In this case, we say that student $i_{t}$ precedes student $i_{t+1}$ in the improvement cycle (with the convention that $i_{k}$ precedes $i_{1}$ ). We say that an assignment $\mu^{\prime}$ is obtained by implementing an improvement cycle at $\mu$ if $\mu^{\prime}$ is obtained from $\mu$ by simply assigning each student in the improvement cycle to the school of the student whom he precedes, keeping the assignments of the students who do not belong to the improvement cycle the same.

Whenever we omit the description of parts of priorities, then they can be completed arbitrarily.

Lemma 7. Suppose that every generalized cycle of the priority profile is tight. Then at each problem where the DA assignment is not efficient, there is a unique efficient Pareto improvement over the DA assignment.

Proof. Let $P=(N, C, R, q, \succeq)$ be a problem such that every generalized cycle of $\left(\succeq_{c}\right)_{c \in C}$ is tight. Let $\mu$ denote the DA assignment.

\footnotetext{
${ }^{21}$ The notion of an improvement cycle is from Dur et al. (2019).

${ }^{22}$ Note that for any $t \in\{1, \ldots, k\},\left(i_{t}, i_{t+1}, \ldots, i_{k}, i_{1}, \ldots, i_{t-1}\right)$ is also an improvement cycle including the same set of students.
} 
Step 1. We first show that there is no student who is included in two different "improvement cycles" at $\mu$.

We claim that any two different improvement cycles cannot have a common student. Suppose not, i.e., $\left(i_{1}, \ldots, i_{k}\right)$ and $\left(j_{1}, \ldots, j_{q}\right)$ are improvement cycles such that $\left(i_{1}, \ldots, i_{k}\right) \neq\left(j_{1}, \ldots, j_{q}\right)$ and $\left\{i_{1}, \ldots, i_{k}\right\} \cap\left\{j_{1}, \ldots, j_{q}\right\} \neq \emptyset$. We claim that there exists $i \in\left\{i_{1}, \ldots, i_{k}\right\} \cap\left\{j_{1}, \ldots, j_{q}\right\}$ such that the student preceding $i$ in the cycle $\left(i_{1}, \ldots, i_{k}\right)$, say student $j$, and the student preceding $i$ in the cycle $\left(j_{1}, \ldots, j_{k}\right)$, say student $j^{\prime}$, are different students, i.e., $j \neq j^{\prime}$. To see this, consider any student $i \in\left\{i_{1}, \ldots, i_{k}\right\} \cap\left\{j_{1}, \ldots, j_{q}\right\}$. If the students preceding $i$ in the two cycles are different, then we are done (as $j \neq j^{\prime}$ ). Otherwise, the student $j$ who precedes $i$ in both cycles satisfies $j \in\left\{i_{1}, \ldots, i_{k}\right\} \cap\left\{j_{1}, \ldots, j_{q}\right\}$. Proceeding similarly, our claim follows from the facts that the two cycles are finite and different.

Now, without loss of generality, suppose that the two cycles are of the form $\left(j, i_{1}, \ldots, i_{k}\right)$ and $\left(j^{\prime}, i_{1}, j_{1}, \ldots, j_{q}\right)$ such that $j \neq j^{\prime}$. Let $c_{1} \equiv \mu\left(i_{1}\right)$. Note that $c_{1} P_{j} \mu(j)$ and $c_{1} P_{j^{\prime}} \mu\left(j^{\prime}\right)$. Then, by the stability of $\mu$, we have $i_{1} \succ_{c_{1}}\left\{j, j^{\prime}\right\}$. Without loss of generality, suppose that $i_{1} \succ_{c_{1}} j^{\prime} \succ_{c_{1}} j$. Let $c_{t} \equiv \mu\left(i_{t}\right)$ for each $t \in\{1, \ldots, k\}$ and let $c_{0} \equiv \mu(j)$. Since $\left(j, i_{1}, \ldots, i_{k}\right)$ is an improvement cycle, by Ergin $(2002)^{23}$ there exist $t \in\{0,1, \ldots, k\}$ and $i \in N \backslash\left\{i_{1}, \ldots, i_{k}, j\right\}$ such that $\left(c_{t}, c_{t-1}, \ldots, c_{0}, c_{k}, c_{k-1}, \ldots, c_{t+1}\right)$ and $\left(i, i_{t-1}, i_{t-2}, \ldots, i_{1}, j, i_{k}, i_{k-1}, \ldots, i_{t+1}, i_{t}\right)$ constitute a cycle of $\left(\succeq_{c}\right)_{c \in C}$ :

\begin{tabular}{cccccccc}
$\succeq_{c_{0}} \succeq_{c_{1}} \succeq_{c_{2}} \succeq_{c_{3}}$ & $\cdots$ & $\succeq_{c_{t}}$ & $\cdots$ & $\succeq_{c_{k}}$ \\
\hline$j$ & $i_{1}$ & $i_{2}$ & $i_{3}$ & & $i_{t}$ & & $i_{k}$ \\
$i_{k}$ & $j^{\prime}$ & $i_{1}$ & $i_{2}$ & $\cdots$ & $i$ & $\cdots$ & $i_{k-1}$ \\
& $j$ & & & & $i_{t-1}$ & &
\end{tabular}

Now $\left(\succeq_{c}\right)_{c \in C}$ includes a generalized cycle that is not tight because (a) for $t \neq 1$, condition I is satisfied, as $i \neq j^{\prime}$ and $i \notin\left\{j, j^{\prime}, i_{1}, \ldots, i_{k}\right\}$, and (b) for $t=1$, condition II is satisfied, as $i \neq j^{\prime}$ and $i \notin\left\{j, j^{\prime}, i_{1}, \ldots, i_{k}\right\}$. This is a contradiction. Hence, no two improvement cycles share a common student.

Step 2. By Lemma 6 of Dur et al. (2019), for any Pareto improvement $\mu^{\prime}$ over the DA assignment $\mu$, there exists a set of disjoint improvement cycles (that is, no two improvement cycles share a common student) such that $\mu^{\prime}$ can be obtained from $\mu$ by implementing the improvement cycles. Since any two improvement cycles are disjoint, there is a unique efficient Pareto improvement over the DA assignment.

Lемма 8. Suppose that every generalized cycle of the priority profile is tight. Then, at each problem, any assignment obtained from the DA assignment by implementing an improvement cycle includes a unique blocking pair.

Proof. Let $P=(N, C, R, q, \succeq)$ be a problem such that every generalized cycle of $\left(\succeq_{c}\right)_{c \in C}$ is tight. Let $\mu$ denote the DA assignment. Let $\mu^{\prime}$ be obtained from $\mu$ by implementing the improvement cycle $\left(i_{1}, \ldots, i_{k}\right)$. Let $c_{t} \equiv \mu\left(i_{t-1}\right)$ for each $t \in\{2, \ldots, k\}$ and $c_{1} \equiv \mu\left(i_{k}\right)$. Suppose that $\mu^{\prime}$ includes two blocking pairs $(i, c) \neq\left(j, c^{\prime}\right)$.

${ }^{23}$ More precisely, it follows from arguments in the proof of Theorem 1 in Ergin (2002). 
Since $\left(i_{1}, \ldots, i_{k}\right)$ is an improvement cycle, by Ergin (2002) there exist $t \in\{1, \ldots, k\}$ (without loss of generality, let $t=1$ ) and $i_{0} \in N \backslash\left\{i_{1}, \ldots, i_{k}\right\}$ such that $\left(c_{1}, \ldots, c_{k}\right)$ and $\left(i_{0}, i_{1}, \ldots, i_{k}\right)$ constitute a cycle of $\left(\succeq_{c}\right)_{c \in C}$ :

$$
\begin{array}{cccc}
\succeq_{c_{1}} \succeq_{c_{2}} & \cdots & \succeq_{c_{k}} \\
\hline i_{k} & i_{1} & \cdots & i_{k-1} \\
i_{0} & i_{2} & \cdots & i_{k} \\
i_{1} & & &
\end{array}
$$

Note that since $(i, c),\left(j, c^{\prime}\right) \in B\left(\mu^{\prime}\right) \backslash B(\mu)$, then $c, c^{\prime} \in\left\{c_{1}, \ldots, c_{k}\right\}$.

Case 1. Suppose that $c=c_{1}$. Since $(i, c) \in B\left(\mu^{\prime}\right) \backslash B(\mu), i_{k} \succ_{c_{1}} i \succ_{c_{1}} i_{1}$. If $i \neq i_{0}$, this contradicts that every generalized cycle is tight. Suppose that $i=i_{0}$. Now either $j \neq i_{0}$ or $c^{\prime} \neq c_{1}$. In either case, by similar arguments, it is easy to see that there is a generalized cycle is that is not tight.

Case 2. Suppose that $c=c_{t}, t \neq 1$. Since $(i, c) \in B\left(\mu^{\prime}\right) \backslash B(\mu)$, then $i_{t-1} \succ_{c_{t}} i \succ_{c_{t}} i_{t}$, which indicates that there is a generalized cycle that is not tight.

Let $P=(N, C, R, q, \succeq)$ be an arbitrary unit-capacity problem with at least five schools such that $\succeq$ satisfies conditions Conditions 1, 2, and 3. By Lemma 6, every generalized cycle of $\left(\succeq_{c}\right)_{c \in C}$ is tight. Then, by Lemma 7 , all the improvement cycles at $\mathrm{DA}(P)$ are distinct and the assignment obtained from $\mathrm{DA}(P)$ by implementing the improvement cycles, let us call it $\mu$, is the unique efficient Pareto improvement over DA $(P)$. Now there can be at most one improvement cycle, since otherwise there must exist two generalized cycles that are either distinct, which would be a violation of Condition 3 by Lemma 4, or distinct except for the initiator, which would be a violation of Condition 2 or 3 by Lemma 5 . Now, by Lemma $8, \mu$ includes at most one blocking pair.

If $\mu$ includes no blocking pair, then it is trivially cardinally minimally unstable among efficient assignments (in fact, this means that the DA assignment is efficient at this problem). Suppose that $\mu$ includes a unique blocking pair. Suppose that $\mu$ is not cardinally minimally unstable among efficient assignments. Then there exist an efficient assignment $\mu^{\prime}$ without a blocking pair. But then $\operatorname{DA}(P)$ does not include any improvement cycle and, therefore, $\mu$ cannot have a blocking pair, a contradiction.

Thus, $\mu=\operatorname{EADA}(P)$ and the EADA mechanism is cardinally minimally unstable among efficient assignments.

\section{STABility COMPARISONS BASED ON BLOCKING STUDENTS}

Another natural stability comparison is based on comparing the sets of blocking students—students involved in at least one blocking pair. Let BS $(\mu)=\left\{i \in N: B_{i}(\mu) \neq \emptyset\right\}$ denote the set of blocking students at $\mu$. The blocking students inclusion comparison (sincl) is defined as follows. For each problem $P \in \mathcal{P}$ and $\mu, \nu \in \mathcal{A}$,

$$
\mu \gtrsim_{\text {sincl }}^{P} \nu \Leftrightarrow \mathrm{BS}(\mu) \subseteq \mathrm{BS}(\nu) .
$$

The blocking students cardinality comparison (scard) is defined as follows. For each $P \in \mathcal{P}$ and $\mu, \nu \in \mathcal{A}(P)$,

$$
\mu \gtrsim_{\text {scard }}^{P} \nu \Leftrightarrow|\mathrm{BS}(\mu)| \leq|\mathrm{BS}(\nu)|
$$


We write BS-wise more stable instead of sincl more stable, BS-wise minimally unstable instead of sincl minimally unstable, BS-wise cardinally more stable instead of scard more stable, and BS-wise cardinally minimally unstable instead of scard minimally unstable. $^{24}$

Interestingly, there is no Pareto improvement over the DA mechanism that is BS-wise minimally unstable among efficient assignments and, in particular, the EADA mechanism is not BS-wise minimally unstable among efficient assignments. An immediate corollary is an impossibility result also with BS-wise cardinal minimal instability. We prove these impossibilities in Section 5. Below we investigate the same questions as in Theorem 1 for BS-wise cardinal minimal instability (instead of cardinal minimal instability). ${ }^{25}$ The corresponding result is almost identical except for one new cycle condition.

We say that a list of three students $\left(i_{1}, i_{2}, i_{3}\right)$ and a pair of schools $\left(c_{1}, c_{2}\right)$ constitute a weakly tight Ergin cycle if they constitute an Ergin cycle, and there is no $m \in N \backslash\left\{i_{1}, i_{2}, i_{3}\right\}$ such that $i_{3} \succ_{c_{1}} m \succ_{c_{1}} i_{2}$ or $i_{2} \succ_{c_{2}} m \succ_{c_{2}} i_{3}{ }^{26}$

Condition 4. All Ergin cycles are weakly tight.

THEOREM 2. Suppose each school has unit capacity and there are at least five schools. The following statements are equivalent:

(i) There exists a mechanism that is both a Pareto improvement over the DA mechanism and BS-wise cardinally minimally unstable among efficient assignments.

(ii) The priority profile $\succeq$ satisfies Conditions 2, 3, and 4 .

(iii) There is a unique mechanism that is both a Pareto improvement over the DA mechanism and BS-wise cardinally minimally unstable among efficient assignments (and, therefore, this mechanism coincides with the EADA mechanism).

The proof of Theorem 2 is similar to the proof of Theorem 1 and is available in Doğan and Ehlers (2020a).

REMARK 5. (a) The proof of Theorem 2 shows that if the priority profile satisfies Conditions 2, 3, and 4, then for each problem, either the DA assignment is efficient or there exists a unique Pareto improvement of the DA assignment that contains exactly one blocking student. Therefore, the latter assignment coincides with EADA assignment.

(b) By Remark 4(a), under Conditions 1, 2, and 3, the EADA assignment contains no blocking student if the DA assignment is efficient and otherwise contains exactly

\footnotetext{
${ }^{24}$ Note that $\gtrsim_{\text {sincl }}^{P} \subseteq \gtrsim_{\text {scard }}^{P}, \gtrsim_{\text {sincl }}^{P}$ is transitive but not complete, and $\gtrsim_{\text {scard }}^{P}$ is complete and transitive.

${ }^{25}$ Note that such a result does not follow directly from Remark 3 because the blocking students cardinality comparison fails the "reasonability" property: we may have $\perp(B(\mu))=(+1,+1),|B(\nu)|>1$ and $\mu \sim \underset{\text { scard }}{P} \nu$ when $\mathrm{BS}(\mu)=\mathrm{BS}(\nu)$.

${ }^{26}$ Note that every tight Ergin cycle is also weakly tight.
} 
one blocking student. Thus, the EADA mechanism is BS-wise cardinally minimally unstable among efficient assignments if the priority profile satisfies Conditions 1 , 2, and 3. However, the priority profile

\begin{tabular}{cc}
$\succeq_{c_{1}} \succeq_{c_{2}}$ \\
\hline 3 & 2 \\
1 & 1 \\
2 & 3
\end{tabular}

satisfies Conditions 2, 3, and 4, but violates Condition 1. Hence, the EADA mechanism is BS-wise cardinally minimally unstable among efficient assignments, but not cardinally minimally unstable among efficient assignments.

(c) Instead of blocking students, one might count "envied schools" (ES). However, in school choice students are agents and schools are objects to be consumed. Now if we count envied schools, there might be only one envied school with many students, whereas there are only two students envying two schools, which might be a counterintuitive comparison in school choice. ${ }^{27}$

\section{Impossibilities}

There exists a mechanism that is a Pareto improvement over the DA mechanism and minimally unstable among efficient assignments (for the blocking pairs inclusion comparison). In fact, we prove a stronger result. Let us call an assignment $\mu$ strongly minimally unstable at $P$ among efficient assignments if $\mu$ is efficient and for any efficient assignment $\nu$ such that $B(\nu) \subseteq B(\mu)$, we have $\mu=\nu$. We show that the EADA mechanism always chooses an assignment that is strongly minimally unstable among efficient assignments. We present our proof in Appendix A.1.

Proposition 1 (Possibility with Minimal Instability). The EADA mechanism is strongly minimally unstable among efficient assignments.

Next we show that all other stability comparisons we have considered result in impossibilities.

Proposition 2 (Impossibilities with Cardinal Minimal Instability). (i) There is no mechanism that is both a Pareto improvement over the DA mechanism and cardinally minimally unstable among efficient assignments. In particular, the EADA mechanism is not cardinally minimally unstable among efficient assignments.

(ii) For any $n \geq 3$, there exists a unit-capacity problem $P$ with $|N|=n$ and $|C|=n-1$, and an efficient assignment $\mu$ such that $\operatorname{EADA}(P)$ is the unique efficient Pareto improvement over $\mathrm{DA}(P)$ and

$$
|B(\operatorname{EADA}(P))|=n-1 \text { and }|B(\mu)|=1 .
$$

\footnotetext{
${ }^{27}$ Nevertheless for envied schools-wise cardinal minimal instability, a result close to Theorem 2 might be obtained due to the fact that in the proof of (ii) $\Rightarrow$ (iii) of Theorem 1 (where $\operatorname{ES}(\mu)=\{c \in C:(i, c) \in$ $B(\mu)$ for some $i \in N\}$, except for Case 3 of Lemma 1, in all the examples we have $\left|\operatorname{ES}\left(\mu^{\prime}\right)\right|=1$ and $|\operatorname{ES}(\mu)|>1$.
} 
(iii) The EADA mechanism is not cardinally minimally unstable among efficient Pareto improvements over the DA mechanism.

(iv) For any $n \geq 5$, there exists a unit-capacity problem $P$ with $|N|=n$ and $|C|=n-1$, and an efficient assignment $\mu$ that Pareto improves over $\mathrm{DA}(P)$ and

$$
|B(\operatorname{EADA}(P))|=n-2 \text { and }|B(\mu)|=2 .
$$

Proof. Note that (i) follows from (ii) and (iii) follows from (iv).

(ii). Consider the following problem $P$ : Let $N=\{1,2,3, \ldots, n\}, C=\left\{c_{1}, c_{2}, c_{3}, \ldots\right.$, $\left.c_{n-1}\right\}$ (all with unit capacities), and

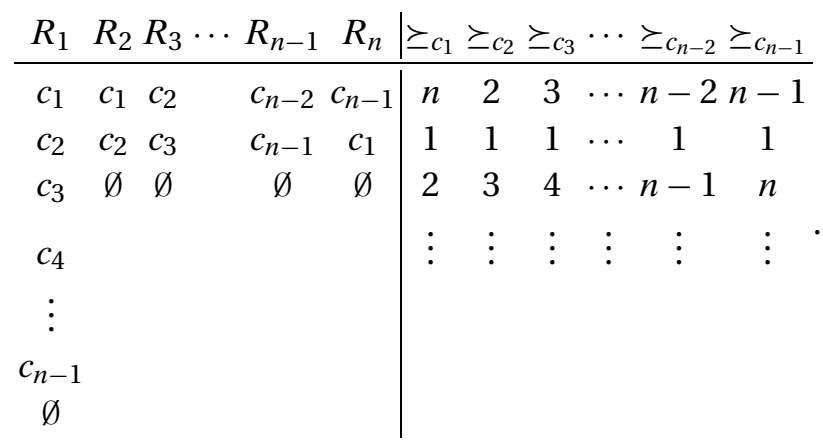

Note that

$$
\begin{aligned}
\operatorname{DA}(P) & =\left(\begin{array}{cccccc}
1 & 2 & 3 & \cdots & n-1 & n \\
\emptyset & c_{2} & c_{3} & \cdots & c_{n-1} & c_{1}
\end{array}\right) \\
\operatorname{EADA}(P) & =\left(\begin{array}{cccccc}
1 & 2 & 3 & \cdots & n-1 & n \\
\emptyset & c_{1} & c_{2} & \cdots & c_{n-2} & c_{n-1}
\end{array}\right)
\end{aligned}
$$

and $B(\operatorname{EADA}(P))=\left\{\left(1, c_{1}\right),\left(1, c_{2}\right), \ldots,\left(1, c_{n-2}\right),\left(1, c_{n-1}\right)\right\}$. Note that the EADA assignment is the unique efficient Pareto improvement over the DA assignment.

Consider the assignment $\mu=\left(\begin{array}{cccccc}1 & 2 & 3 & \cdots & n-1 & n \\ c_{1} & c_{2} & c_{3} & \cdots & c_{n-1} & \emptyset\end{array}\right)$, where $B(\mu)=\left\{\left(n, c_{1}\right)\right\}$. Note that $\mu$ is efficient and is cardinally more stable than $\operatorname{EADA}(P)$.

(iv). Consider the following problem $P$ : Let $N=\{1,2,3, \ldots, n\}, C=\left\{c_{1}, c_{2}, c_{3}, \ldots\right.$, $\left.c_{n-1}\right\}$ (all with unit capacities), and

\begin{tabular}{ccccccc|cccccccc}
$R_{1}$ & $R_{2}$ & $R_{3}$ & $\cdots$ & $R_{n-2}$ & $R_{n-1}$ & $R_{n}$ & $\succeq_{c_{1}}$ & $\succeq_{c_{2}} \succeq_{c_{3}}$ & $\cdots$ & $\succeq_{c_{n-3}} \succeq_{c_{n-2}} \succeq_{c_{n-1}}$ \\
\hline$c_{1}$ & $c_{1}$ & $c_{2}$ & $\cdots$ & $c_{n-3}$ & $c_{1}$ & $c_{n-1}$ & $n$ & 2 & 3 & $\cdots$ & $n-3$ & 1 & $n-2$ \\
$c_{2}$ & $c_{2}$ & $c_{3}$ & $\cdots$ & $c_{1}$ & $\emptyset$ & $c_{n-2}$ & $n-1$ & 1 & 1 & $\cdots$ & 1 & $n$ & $n$ \\
$c_{3}$ & $\emptyset$ & $\emptyset$ & $\cdots$ & $c_{n-1}$ & & $c_{1}$ & $n-2$ & 3 & 4 & $\cdots$ & $n-2$ & $\vdots$ & 1 \\
$\vdots$ & & & $\emptyset$ & & $\emptyset$ & $\vdots$ & $\vdots$ & $\vdots$ & $\cdots$ & $\vdots$ & & $\vdots$ \\
$c_{n-3}$ & & & & & & 3 & & & & & & \\
$c_{n-2}$ & & & & & & 1 & & & & & \\
$\varnothing$ & & & & & & 2 & & & & &
\end{tabular}.


Note that

$$
\begin{aligned}
\operatorname{DA}(P) & =\left(\begin{array}{ccccccc}
1 & 2 & 3 & \cdots & n-2 & n-1 & n \\
c_{n-2} & c_{2} & c_{3} & \cdots & c_{n-1} & \emptyset & c_{1}
\end{array}\right) \\
\operatorname{EADA}(P) & =\left(\begin{array}{ccccccc}
1 & 2 & 3 & \cdots & n-2 & n-1 & n \\
c_{n-2} & c_{1} & c_{2} & \cdots & c_{n-3} & \emptyset & c_{n-1}
\end{array}\right)
\end{aligned}
$$

$B(\operatorname{EADA}(P))=\left\{\left(1, c_{1}\right),\left(1, c_{2}\right), \ldots,\left(1, c_{n-3}\right),\left(n-1, c_{1}\right)\right\}$, and $|B(\operatorname{EADA}(P))|=n-2$.

Consider the assignment

$$
\mu=\left(\begin{array}{ccccccc}
1 & 2 & 3 & \cdots & n-2 & n-1 & n \\
c_{1} & c_{2} & c_{3} & \cdots & c_{n-1} & \emptyset & c_{n-2}
\end{array}\right),
$$

where $B(\mu)=\left\{\left(n-2, c_{1}\right),\left(n-1, c_{1}\right)\right\}$ and $|B(\mu)|=2$. Note that $\mu$ is efficient, $\mu$ Pareto improves $\mathrm{DA}(P)$, and $\mu$ is cardinally more stable than $\operatorname{EADA}(P)$.

Below we show that Proposition 1 does not extend to BS-wise minimal instability.

Proposition 3 (Impossibilities with BS-wise Minimal Instability). (i) There is no mechanism that is both a Pareto improvement over the DA mechanism and BS-wise minimally unstable among efficient assignments. In particular, the EADA mechanism is not BS-wise minimally unstable among efficient assignments.

(ii) The EADA mechanism is not BS-wise minimally unstable among efficient Pareto improvements over the DA mechanism.

Proof. We show both (i) and (ii) by considering the following problem $P$ : Let $N=$ $\{1,2,3,4,5,6,7\}, C=\left\{c_{1}, c_{2}, c_{3}, c_{4}, c_{5}, c_{6}\right\}$ (all with unit capacities), and

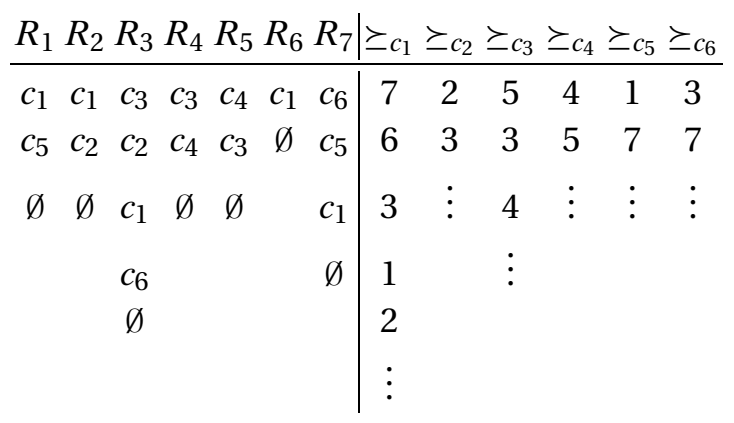

Note that

$$
\begin{aligned}
\operatorname{DA}(P) & =\left(\begin{array}{ccccccc}
1 & 2 & 3 & 4 & 5 & 6 & 7 \\
c_{5} & c_{2} & c_{6} & c_{4} & c_{3} & \emptyset & c_{1}
\end{array}\right) \\
\operatorname{EADA}(P) & =\left(\begin{array}{ccccccc}
1 & 2 & 3 & 4 & 5 & 6 & 7 \\
c_{5} & c_{1} & c_{2} & c_{3} & c_{4} & \emptyset & c_{6}
\end{array}\right)
\end{aligned}
$$

and $\operatorname{BS}(\operatorname{EADA}(P))=\{1,3,6\}$ (since $\left.B(\operatorname{EADA}(P))=\left\{\left(1, c_{1}\right),\left(3, c_{3}\right),\left(6, c_{1}\right)\right\}\right)$. 
Consider the assignment

$$
\mu=\left(\begin{array}{ccccccc}
1 & 2 & 3 & 4 & 5 & 6 & 7 \\
c_{1} & c_{2} & c_{6} & c_{3} & c_{4} & \emptyset & c_{5}
\end{array}\right),
$$

where $\mathrm{BS}(\mu)=\{3,6\}$ (since $B(\mu)=\left\{\left(3, c_{1}\right),\left(6, c_{1}\right)\right\}$ ). Note that $\mu$ is efficient and Pareto improves over DA. Moreover, $\mu$ is BS-wise more stable than $\operatorname{EADA}(P)$ since $\mathrm{BS}(\mu) \subsetneq$ $\operatorname{BS}(\operatorname{EADA}(P))$. Furthermore, note that $\mu$ and $\operatorname{EADA}(P)$ are the only efficient Pareto improvements over $\mathrm{DA}(P) .^{28}$

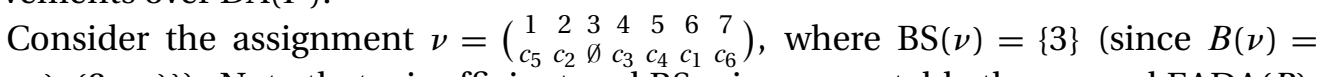
$\left.\left\{\left(3, c_{3}\right),\left(3, c_{6}\right)\right\}\right)$. Note that $\nu$ is efficient and BS-wise more stable than $\mu$ and $\operatorname{EADA}(P)$.

Since BS-wise cardinal minimal instability implies BS-wise minimal instability, Proposition 3 remains unchanged if we replace BS-wise minimal instability with BS-wise cardinal minimal instability.

\section{Discussion}

There is another important property for the design of mechanisms that we have not discussed so far: strategy-proofness. A mechanism is strategy-proof if at any problem, no student can be better off by reporting a preference relation different than his true preference relation. Proposition 1 in Kesten (2010) shows that there is no efficient and strategy-proof mechanism that selects the efficient and stable assignment whenever it exists. Hence, there exists no mechanism that is strategy-proof and minimally unstable among efficient assignments for any of our stability comparisons based on blocking pairs or blocking students. Even if the priority profile satisfies Conditions 1, 2, and 3 and the EADA mechanism does not coincide with DA, the EADA mechanism is not strategyproof: this follows from Abdulkadiroğlu et al. (2009).

The top trading cycles (TTC) mechanism (Abdulkadiroğlu and Sönmez (2003)), which is based on Gale's TTC algorithm (Shapley and Scarf (1974)), is another well known efficient mechanism. The TTC mechanism is not a Pareto improvement over the DA mechanism, but it is strategy-proof. The TTC mechanism is not minimally unstable among efficient assignments simply because, TTC may not choose the efficient and stable assignment when it exists. Yet, Abdulkadiroğlu et al. (2020) has shown that if we fix a set of agents and a set of schools with unit capacities, then TTC is minimally unstable among efficient and strategy-proof mechanisms. In our companion paper Doğan and Ehlers (2020b), we show that TTC is also minimally unstable among efficient and strategy-proof mechanisms for any of our stability comparisons if we fix a set of agents and a set of schools with unit capacities.

Another known efficient mechanism is the top trading cycles over DA mechanism (DA $\oplus$ TTC), which is based on applying Gale's TTC procedure over the DA outcome,

\footnotetext{
${ }^{28}$ This follows because for any efficient Pareto improvment $\eta$ over $\mathrm{DA}(P)$, we must have $\eta(6)=\emptyset, \eta(4)=$ $c_{3}, \eta(5)=c_{4}$, and $\eta(7) \neq c_{1}$. If $\eta(7)=c_{5}$, then $\eta=\mu$ and if $\eta(7)=c_{6}$, then $\eta=\mu$.
} 
i.e., first run the DA algorithm and obtain the DA assignment; then, using the DA assignment as the endowment profile, calculate the TTC assignment as in Shapley and Scarf (1974). The DA $\oplus$ TTC mechanism is efficient and Pareto improves over DA. The $\mathrm{DA} \oplus \mathrm{TTC}$ assignments are also not necessarily minimally unstable among efficient assignments, since there exist problems where the EADA assignment is more stable (see Example 8 in Kesten (2010)). Hence, DA $\oplus$ TTC is also not cardinally minimally unstable among efficient assignments, which also follows from Proposition 3.

Furthermore, stability is equivalent to the conjunction of non-wastefulness and no justified envy. ${ }^{29}$ Now any efficient assignment is non-wasteful, and for efficient assignments, stability is equivalent to no justified envy. Thus, the concepts of (cardinal) minimal instability are equivalent to (cardinal) minimal justified envy. ${ }^{30}$

Another alternative stability comparison is based on blocking triplets. Kwon and Shorrer (2019) study the blocking triplets inclusion comparison (tincl), which is defined (given problem $P$ and assignment $\mu$ ) as $(i, j, c) \in T(\mu)$ if and only if $i \succ_{c} j, \mu(j)=c$, and $c P_{i} \mu(i)$; then for $\mu, \nu \in \mathcal{A}$,

$$
\mu \gtrsim_{\text {tincl }}^{P} \nu \Leftrightarrow T(\mu) \subseteq T(\nu) .
$$

Now the blocking triplets cardinality comparison tcard is defined, for each $P \in \mathcal{P}$ and $\mu, \nu \in \mathcal{A}$, as

$$
\mu \gtrsim_{\text {tcard }}^{P} \nu \Leftrightarrow|T(\mu)| \leq|T(\nu)|
$$

Note that $\gtrsim_{\text {tincl }}^{P} \subseteq \gtrsim_{\text {tcard }}^{P}$.

An immediate observation is that Theorem 1 extends to the blocking triplets cardinality comparison tcard. As for problems where schools have unit capacity, for any problem $P$, we have $|B(\mu)|=|T(\mu)|$ for any efficient assignment $\mu$. Hence, tcard and scard coincide on the set of efficient assignments. This is due to the fact that efficient assignments are non-wasteful, and stability and no justified envy become equivalent. The same is true for Propositions 2 and 3, as in the examples of the proofs, all schools have unit capacities. ${ }^{31}$

${ }^{29}$ Given problem $P$, (i) an assignment $\mu$ is non-wasteful if there exists no $(i, c) \in B(\mu)$ with $|\mu(c)|<q_{c}$, and (ii) an assignment $\mu$ has no justified envy if there exists no $(i, c) \in B(\mu)$ with $|\mu(c)|=q_{c}$.

${ }^{30}$ This is the terminology used by Abdulkadiroğlu et al. (2020) and Kwon and Shorrer (2019). In a recent paper, Romm et al. (2020) show that in different contexts, the concepts of blocking and justified envy may diverge.

${ }^{31}$ Furthermore, strong minimal instability of EADA a.e.a. that we prove in Appendix A.1 also holds for tincl and implies Proposition 4 of Kwon and Shorrer (2019): Suppose, by contradiction, that there are a problem $P, \nu=\operatorname{EADA}(P)$, and an efficient assignment $\mu$ such that $\mu \gtrsim_{\text {tincl }}^{P} \nu$ and $\mu \neq \nu$; then by definition $T(\mu) \subseteq T(\nu)$, and [I] any $(i, j, c) \in T(\mu)$ implies $(i, c) \in B(\mu)$ and [II] any $(i, j, c) \in T(\nu)$ implies $(i, c) \in B(\nu)$. Since $B(\mu)=\{(i, c):(i, j, c) \in T(\mu)$ for some $j \in N\}$, we obtain $B(\mu) \subseteq B(\nu)$ and $\mu \gtrsim_{\text {pincl }}^{P} \nu$. Now, as shown in the proof of Proposition 1, there exists a unit-capacity problem $P^{\prime}$ and an efficient assignment $\mu^{\prime}$ such that $\mu^{\prime} \gtrsim P_{\text {tincl }}^{\prime} \operatorname{EADA}\left(P^{\prime}\right)$ and $\mu^{\prime} \neq \operatorname{EADA}\left(P^{\prime}\right)$, which implies that $\mu^{\prime} \gtrsim_{\text {pincl }}^{P^{\prime}} \operatorname{EADA}\left(P^{\prime}\right)$, which contradicts Proposition 1 . 


\section{APPENDIX}

\section{A.1 Minimal instability of EADA}

We prove Proposition 1: the EADA mechanism is minimally unstable among efficient assignments. The following notation is useful. Given a set of students $I \subseteq N$ and a set of school $S \subseteq C$, let $\mu(I)=\bigcup_{i \in I} \mu(i)$ and $\mu(S)=\bigcup_{c \in S} \mu(c)$ denote the aggregate assignments of $I$ and $S$ at $\mu$, respectively. Given a set of students $I \subseteq N$, let $\left.\mu\right|_{I}$ denote the restriction of $\mu$ to $I$. Note that $\left.\mu\right|_{I}$ is a mapping $\left.\mu\right|_{I}: I \cup \mu(I) \rightarrow I \cup \mu(I)$.

The following results from the literature are useful. The following lemma is Lemma 2 of Tang and $\mathrm{Yu}$ (2014), which shows that each step of the EADA algorithm Pareto improves upon the previous step.

Lemma 9. For each $t \in\{2, \ldots, m\}, \mu_{t}$ Pareto improves $\mu_{t-1}$. Also, $\mu_{1}$ Pareto improves the DA assignment.

The following lemma follows from Doğan and Yenmez (2020), which shows that the EADA satisfies a particular consistency property.

LEMMA 10. Whenever a student who is assigned to a school that is underdemanded at DA is removed with his assigned seat at the EADA assignment, the assignments of the remaining students do not change when the EADA algorithm is run for the reduced problem.

The proof is in two steps. First, we prove the statement for problems at which each school has unit capacity. Then we extend it to the entire domain of problems.

Unit-capacity case. Let $P=(N, C, R, q, \succeq)$ be a problem such that for each $c \in C$, $q_{c}=1$. Let $\mu=\operatorname{EADA}(P)$. Let $\left\{I_{1}, \ldots, I_{m}\right\}$ denote the partition of $N$ generated by the underdemanded schools algorithm. Let $\nu=\mathrm{DA}(P)$. For each $t \in\{1, \ldots, m\}$, let $I_{>t}=I_{t+1} \cup \cdots \cup I_{m}$ (and, similarly, we define $I_{\leq t}$ and $I_{<t}$ ). Let $\mu_{1}, \ldots, \mu_{m}$ be as defined above, i.e., for each $t \in\{1, \ldots, m\}, \mu_{t}$ is the assignment obtained by iterating the EADA algorithm for only $t$ rounds.

We first claim that $B\left(\mu_{1}\right) \subseteq B\left(\mu_{2}\right) \subseteq \cdots \subseteq B\left(\mu_{m}\right)=B(\mu)$. Note that for each $t \in$ $\{1, \ldots, m-1\}, \mu_{t+1}$ is a Pareto improvement over $\mu_{t}$ by Lemma 9 . Then, for each school $c \in C$, the student who is assigned to $c$ in $\mu_{t+1}$ has a weakly lower priority than the student who is assigned to $c$ in $\mu_{t}$, since otherwise $\mu_{t}$ restricted to $I_{\geq t}$ would be unstable. Thus, for each student $i \in I_{\leq t}$, the set of blocking pairs in $\mu_{t}$ that include $i$ is a subset of the set of the set of blocking pairs in $\mu_{t+1}$ that include $i$. Moreover, since no student in $I_{>t}$ prefers a school that is assigned to a student in $I_{\leq t}$ to his assigned school in $\mu_{t+1}$, no student in $I_{\geq t+1}$ is included in a blocking pair in $\mu_{t}$ or $\mu_{t+1}$, which proves the claim.

Suppose to the contrary that $\mu^{\prime}$ is an efficient assignment such that $B\left(\mu^{\prime}\right) \subseteq B(\mu)$ and $\mu^{\prime} \neq \mu$. Then, for some $j \in N, \mu^{\prime}(j) \neq \mu(j)$. Let $j \in I_{t}$. If $\mu\left(I_{t}\right)=\mu^{\prime}\left(I_{t}\right)$, then by the underdemanded schools algorithm, $\mu(i)=\mu_{t}(i)$ for all $i \in I_{t}$. But then $B_{i}(\mu) \cap\left(I_{t} \times\right.$ $\left.\mu\left(I_{t}\right)\right)=\emptyset$ for all $i \in I_{t}$. By $B\left(\mu^{\prime}\right) \subseteq B(\mu)$, we also have $B_{i}\left(\mu^{\prime}\right) \cap\left(I_{t} \times \mu\left(I_{t}\right)\right)=\emptyset$ for all $i \in I_{t}$. Thus, both $\left.\mu\right|_{I_{t}}$ and $\left.\mu^{\prime}\right|_{I_{t}}$ are stable. Because $\mu\left(I_{t}\right)=\mu^{\prime}\left(I_{t}\right)$, it now follows from efficiency that $\mu(i)=\mu^{\prime}(i)$ for all $i \in I_{t}$, a contradiction to $\mu^{\prime}(j) \neq \mu(j)$ and $j \in I_{t}$. Thus, $\mu\left(I_{t}\right) \neq \mu^{\prime}\left(I_{t}\right)$. 
Suppose, without loss of generality, that $\mu^{\prime}(j) \notin \mu\left(I_{t}\right)$. But then going from $\mu$ to $\mu^{\prime}$ agent $j$ is involved in a trading cycle $j_{1}, \ldots, j_{r}$ such that $\mu^{\prime}\left(j_{l}\right)=\mu\left(j_{l+1}\right)$ for each $l \in\{1, \ldots, r-1\}$, and $\mu^{\prime}\left(j_{r}\right)=\mu\left(j_{1}\right)$. Let $c_{l}=\mu\left(j_{l}\right)$ for each $l \in\{1, \ldots, r\}$.

We show that there exists $l \in\{1, \ldots, r\}$ such that $j_{l} \in I_{t}, c_{l+1} \in \mu\left(I_{>t}\right)$, and $c_{l+1} P_{j_{l}} c_{l}$. By efficiency of $\mu^{\prime}$, there exists $l \in\{1, \ldots, r\}$ such that $c_{l+1} P_{j_{l}} c_{l}$. Then by the underdemanded schools algorithm, $c_{l+1} \in \mu\left(I_{\geq t}\right)$. If $c_{l+1} \in \mu\left(I_{>t}\right)$, then we have the desired $l$. Suppose not, that is, suppose that $c_{l+1} \in \mu\left(I_{t}\right)$. Then we have $\mu_{t}\left(j_{l}\right)=\mu\left(j_{l}\right)=c_{l}$ and $\mu_{t}\left(j_{l+1}\right)=\mu\left(j_{l+1}\right)=c_{l+1}$ which implies $j_{l+1} \succ_{c_{l+1}} j_{l}$. Since $\left(j_{l+1}, c_{l+1}\right) \notin B(\mu)$ and $B\left(\mu^{\prime}\right) \subseteq B(\mu)$, we have $\left(j_{l+1}, c_{l+1}\right) \notin B\left(\mu^{\prime}\right)$ and $c_{l+2}=\mu^{\prime}\left(j_{l+1}\right) P_{j_{l+1}} c_{l+1}$. But then again $c_{l+2} \in \mu\left(I_{\geq t}\right)$. If $c_{l+2} \in \mu\left(I_{>t}\right)$, we have the desired $l$. Otherwise, $c_{l+2} \in \mu\left(I_{t}\right)$. Since $j \in\left\{j_{1}, \ldots, j_{r}\right\}$ and $\mu^{\prime}(j) \notin \mu\left(I_{t}\right)$, by continuing similarly we eventually reach the desired $l$.

Now, without loss of generality, let $j$ be such that $j \in I_{t}, \mu^{\prime}(j) \in \mu\left(I_{>t}\right)$, and $\mu^{\prime}(j) P_{j} \mu(j)$. Let $i_{1}=\mu_{t}\left(\mu^{\prime}(j)\right)$. Note that $i_{1} \in I_{>t}$ since $\mu^{\prime}(j) \in \mu\left(I_{>t}\right)$. By $\mu^{\prime}(j) P_{j} \mu(j)$, we have $i_{1} \succ_{\mu^{\prime}(j)} j$. Thus, $\mu\left(i_{1}\right) R_{i_{1}} \mu^{\prime}(j)$ and $\left(i_{1}, \mu^{\prime}(j)\right) \notin B(\mu)$. By $B\left(\mu^{\prime}\right) \subseteq B(\mu),\left(i_{1}, \mu^{\prime}(j)\right) \notin$ $B\left(\mu^{\prime}\right)$. Hence, $\mu^{\prime}\left(i_{1}\right) P_{i_{1}} \mu^{\prime}(j)$ and since $i_{1} \in I_{>t}$, by the underdemanded schools algorithm, $\mu^{\prime}\left(i_{1}\right) \in \mu\left(I_{>t}\right)$.

Let $i_{2}=\mu_{t}\left(\mu^{\prime}(j)\right)$. By similar arguments, there exists an agent $i_{3}$ such that $i_{3}=$ $\mu_{t}\left(\mu^{\prime}\left(i_{2}\right)\right), \mu^{\prime}\left(i_{3}\right) \in \mu\left(I_{>t}\right)$, and $\mu^{\prime}\left(i_{3}\right) P_{i_{3}} \mu\left(i_{3}\right)$. Continuing similarly, since the number of agents is finite, we eventually reach an agent $i_{r}$ such that $j=\mu_{t}\left(\mu^{\prime}\left(i_{r}\right)\right), \mu^{\prime}\left(i_{r}\right) \in \mu\left(I_{>t}\right)$, and $\mu^{\prime}\left(i_{r}\right) P_{i_{r}} \mu\left(i_{r}\right)$, which is a contradiction since $\mu^{\prime}\left(i_{r}\right)=\mu(j)$ and $\mu(j) \in \mu\left(I_{t}\right)$.

Extension to general capacities. Let $P=(N, C, R, q, \succeq)$ be a problem (not necessarily unit capacity). We construct an auxiliary unit-capacity problem $P^{\prime}=\left(N, C^{\prime}, R^{\prime}, q^{\prime}, \succeq^{\prime}\right)$, which has the same set of students, as follows.

(i) For each $c \in C$, we construct $q_{c}$ unit-capacity schools labelled as $c_{1}, \ldots, c_{q_{c}}$ and assign them into $C^{\prime}$. Note that $\left|C^{\prime}\right|=\sum_{c \in C} q_{c}$ and $q^{\prime}$ is a $\left|C^{\prime}\right|$-tuple of 1 s.

(ii) For each $i \in N$ and $c_{p}, c_{q}^{\prime} \in C^{\prime}$, we have $c_{p} R_{i}^{\prime} c_{q}^{\prime}$ if and only if $c P_{i} c^{\prime}$ or $\left[c=c^{\prime}\right.$ and $p \leq q$ ]. Also, $c_{p} P_{i} i$ if and only if $c P_{i} i$.

(iii) For each $c_{p} \in C^{\prime}$ and $i, j \in N$, we have $i \succeq_{c_{p}}^{\prime} j$ if and only if $i \succeq_{c} j$. Also, $i \succeq_{c_{p}} c_{p}$ if and only if $i \succeq_{c} c$.

We define a mapping $\varphi$ from the set of assignments in $P^{\prime}$ to the set of assignments in $P$ as follows. Given an assignment $\mu$ in problem $P^{\prime}$, let $\varphi\left(\mu^{\prime}\right)$ be the assignment in problem $P$ such that for each $i \in N, \varphi\left(\mu^{\prime}\right)(i)=c$ if and only if $\mu^{\prime}(i)=c_{p}$ for some $p \in$ $\left\{1, \ldots, c_{q_{c}}\right\}$.

Observation 1. $\varphi\left(\mathrm{DA}\left(P^{\prime}\right)\right)=\mathrm{DA}(P)$. To see this, first note that clearly $\varphi\left(\mathrm{DA}\left(P^{\prime}\right)\right)$ is stable at $P$. Suppose that $\varphi\left(\mathrm{DA}\left(P^{\prime}\right)\right) \neq \mathrm{DA}(P)$. Since $\mathrm{DA}(P)$ is the student-optimal stable assignment at $P, \mathrm{DA}(P)$ Pareto improves $\varphi\left(\mathrm{DA}\left(P^{\prime}\right)\right)$. Let $\mu$ be the assignment in problem $P^{\prime}$ such that for each $i \in N, \mu(i)=c_{p}$ if and only if $\operatorname{DA}(P)(i)=c$ and $\mid j \in \operatorname{DA}(P)(c): j \succeq_{c}$ $i \mid=p$. Note that $\mu$ is stable and Pareto improves $\mathrm{DA}\left(P^{\prime}\right)$ at $P^{\prime}$, which is a contradiction since $\operatorname{DA}\left(P^{\prime}\right)$ is the student-optimal stable assignment at $P^{\prime}$. 
Observation 2. Each student $i \in N$ who is assigned to an underdemanded school at $\mathrm{DA}\left(P^{\prime}\right)$ is assigned to an underdemanded school also at $\mathrm{DA}(P)$. (Note that it is not necessarily true that if $c \in C$ is underdemanded at DA $(P)$, each of $c_{1}, \ldots, c_{q_{c}}$ is underdemanded at $\mathrm{DA}\left(P^{\prime}\right)$.) This easily follows from $\varphi\left(\mathrm{DA}\left(P^{\prime}\right)\right)=\mathrm{DA}(P)$.

We show that $\varphi\left(\operatorname{EADA}\left(P^{\prime}\right)\right)=\operatorname{EADA}(P)$. Let $i_{1} \in N$ be a student who is assigned to an underdemanded school $c_{q}$ at DA $\left(P^{\prime}\right)$ (Since all the students who are assigned to a school at the last step of the DA algorithm are assigned to underdemanded schools, there exists such a student). By Observation 1, $\varphi\left(\mathrm{DA}\left(P^{\prime}\right)\right)\left(i_{1}\right)=\mathrm{DA}(P)(i)=c$. By Observation 2, $c$ is underdemanded at $\mathrm{DA}(P)$. Then $\varphi\left(\operatorname{EADA}\left(P^{\prime}\right)\right)\left(i_{1}\right)=\operatorname{EADA}(P)\left(i_{1}\right)$.

By Lemma 10, at the problem $P^{\prime}$, if student $i_{1}$ is removed with his assigned seat at the $\operatorname{EADA}\left(P^{\prime}\right)$ assignment, the assignments of the remaining students do not change when the EADA algorithm is run for the reduced problem. Again by Lemma 10, at the problem $P$, if student $i$ is removed with his assigned seat at the $\operatorname{EADA}(P)$ assignment, the assignments of the remaining students do not change when the EADA algorithm is run for the reduced problem.

Now we proceed likewise with the reduced problems. Let $i_{2} \in N \backslash\left\{i_{1}\right\}$ be a student who is assigned to an underdemanded school $c_{q}$ at the DA assignment for the problem reduced from $P^{\prime}$. (As long as $N \backslash\left\{i_{1}\right\} \neq \emptyset$, there exists such a student.) By similar arguments as above, $\varphi\left(\operatorname{EADA}\left(P^{\prime}\right)\right)\left(i_{2}\right)=\operatorname{EADA}(P)\left(i_{2}\right)$. Proceeding likewise, we eventually exhaust all the students, which concludes that $\varphi\left(\operatorname{EADA}\left(P^{\prime}\right)\right)=\operatorname{EADA}(P)$.

Suppose that $\operatorname{EADA}(P)$ is not minimally unstable among efficient assignments. Let $\mu \neq \operatorname{EADA}(P)$ be an efficient assignment that is weakly more stable than $\operatorname{EADA}(P)$ at $P$. Let $\mu^{\prime}$ be the assignment in problem $P^{\prime}$ such that for each $i \in N, \mu(i)=c_{p}$ if and only if $\operatorname{DA}(P)(i)=c$ and $\left|\left\{j \in \operatorname{DA}(P)(c): j \succeq_{c} i\right\}\right|=p$. Note that $\mu^{\prime} \neq \mu$ is efficient and weakly more stable than $\operatorname{EADA}\left(P^{\prime}\right)$, which is a contraction since the $\mathrm{EADA}$ assignment is minimally unstable among efficient assignments when each school has unit capacity.

\section{A.2 Necessary conditions in the multicapacity case}

A capacity-priority profile $\left(q_{c}, \succeq_{c}\right)_{c \in C}$ includes an (multi-capacity) Ergin cycle (Ergin (2002)) if there exist a list of three students $\left(i_{1}, i_{2}, i_{3}\right)$, a pair of schools $\left(c_{1}, c_{2}\right)$, and a pair of (possibly empty) disjoint sets of students $\left(N_{c_{1}}, N_{c_{2}}\right)$ such that such that $i_{3} \succ_{c_{1}} i_{1} \succ_{c_{1}} i_{2}$ and $i_{2} \succ_{c_{2}} i_{3}$.

i. We have $i_{3} \succ_{c_{1}} i_{1} \succ_{c_{1}} i_{2}$ and $i_{2} \succ_{c_{2}} i_{3}$.

ii. For each $t \in\{1,2\}, N_{c_{t}} \subset N \backslash\left\{i_{1}, i_{2}, i_{3}\right\}$ and $\left|N_{c_{t}}\right|=q_{c_{t}}-1$.

iii. We have $N_{c_{1}} \subset\left\{i \in N: i \succ_{c_{1}} i_{1}\right\}$ and $N_{c_{2}} \subset\left\{i \in N: i \succ_{c_{2}} i_{3}\right\}$.

Given an Ergin cycle consisting of $(1,2,3),\left(c_{1}, c_{2}\right)$, and $\left(N_{c_{1}}, N_{c_{2}}\right)$, we call the first student, student $i_{1}$, the initiator of the Ergin cycle.

Given an Ergin cycle consisting of $\left(i_{1}, i_{2}, i_{3}\right),\left(c_{1}, c_{2}\right)$, and $\left(N_{c_{1}}, N_{c_{2}}\right)$, and another Ergin-cycle consisting of $\left(j_{1}, j_{2}, j_{3}\right),\left(c_{1}^{\prime}, c_{2}^{\prime}\right)$, and $\left(N_{c_{1}^{\prime}}^{\prime}, N_{c_{2}^{\prime}}^{\prime}\right)$, we say that the two generalized cycles are distinct if all the students, schools, and sets of students in the two generalized cycles are distinct; we say that the two generalized cycles are distinct except for 
the initiator if $i_{1}=j_{1}$ and all the other students, schools, and sets of students in the two generalized cycles are distinct.

We say that a list $\left(i_{1}, i_{2}, i_{3}\right),\left(c_{1}, c_{2}\right)$, and $\left(N_{c_{1}}, N_{c_{2}}\right)$ constitute a tight Ergin cycle if they constitute an Ergin cycle and

iv. there is no $m \in N \backslash\left(N_{c_{1}} \cup N_{c_{2}} \cup\left\{i_{2}, i_{3}\right\}\right)$ such that $i_{2} \succ_{c_{2}} m \succ_{c_{2}} i_{3}$

v. and there is no $m \in N \backslash\left(N_{c_{1}} \cup N_{c_{2}} \cup\left\{i_{1}, i_{2}, i_{3}\right\}\right)$ such that $i_{3} \succ_{c_{1}} m \succ_{c_{1}} i_{2}$.

Condition $1^{*}$. All the Ergin cycles are tight.

Condition 2*. There are no two Ergin cycles that are distinct except for the initiator.

Condition $3^{*}$. For any two distinct Ergin cycles consisting of $\left(i_{1}, i_{2}, i_{3}\right),\left(c_{1}, c_{2}\right)$, and $\left(N_{c_{1}}, N_{c_{2}}\right)$, and $\left(j_{1}, j_{2}, j_{3}\right),\left(c_{1}^{\prime}, c_{2}^{\prime}\right)$, and $\left(N_{c_{1}^{\prime}}^{\prime}, N_{c_{2}^{\prime}}^{\prime}\right)$, there does not exist any student $i \in$ $\left\{i_{1}, i_{2}, i_{3}\right\}$, any school $c \in C \backslash\left\{c_{1}, c_{2}, c_{1}^{\prime}, c_{2}^{\prime}\right\}$, and any set of students $N_{c}$ distinct from the students in the two Ergin cycles (including the sets of students in the two Ergin cycles) such that $N_{c} \subset\left\{j \in N: j \succ_{c} i\right\}$ and $\left|N_{c}\right|=q_{c}-1$.

Proposition 4. Suppose that $\left(q_{c}, \succeq_{c}\right)_{c \in C}$ violates Condition $1^{*}$, i.e., it includes an Ergin cycle that is not tight. Then there exists a problem such that there is no Pareto improvement over DA that is cardinally minimally unstable among efficient assignments.

Proof. The proof follows from the same arguments as in the proof of Lemma 1 with the following modifications.

- In the preference profile $R$, for each $t \in\{1,2\}$, each student in $N_{c_{t}}$ top ranks $c_{t}$.

- In the assignments $\mu$ and $\mu^{\prime}$, for each $t \in\{1,2\}$, each student in $N_{c_{t}}$ is assigned to $c_{t}$.

Proposition 5. Suppose that $\left(q_{c}, \succeq_{c}\right)_{c \in C}$ violates Condition $2^{*}$, i.e., it includes two Ergin cycles that are distinct except for the initiator. Then there exists a problem such that there is no Pareto improvement over DA that is cardinally minimally unstable among efficient assignments.

Proof. The proof follows from the same arguments as in the proof of Lemma 2 with the following modifications.

- In the preference profile $R$, for each $t \in\{1,2\}$, each student in $N_{c_{t}}$ top ranks $c_{t}$ and each student in $N_{c_{t}^{\prime}}^{\prime}$ top ranks $c_{t}^{\prime}$.

- In the assignments $\mu$ and $\mu^{\prime}$, for each $t \in\{1,2\}$, each student in $N_{c_{t}}$ is assigned to $c_{t}$ and each student in $N_{c_{t}^{\prime}}^{\prime}$ is assigned to $c_{t}^{\prime}$.

Proposition 6. Suppose that $\left(q_{c}, \succeq_{c}\right)_{c \in C}$ violates Condition $3^{*}$. Then there exists a problem such that there is no Pareto improvement over DA that is cardinally minimally unstable among efficient assignments. 
Proof. The proof follows from the same arguments as in the proof of Lemma 3 with the following modifications.

- Note that in the proof of Lemma 3, existence of a school $c$ with the desired property follows from the unit-capacity assumption and the assumption that $|C| \geq 5$. Here, it directly follows from the violation of $\mathrm{C}^{*}$.

- In the preference profile $R$, each student in $N_{c}$ top ranks $c$, and for each $t \in\{1,2\}$, each student in $N_{c_{t}}$ top ranks $c_{t}$ and each student in $N_{c_{t}^{\prime}}^{\prime}$ top ranks $c_{t}^{\prime}$.

- In the assignments $\mu$ and $\mu^{\prime}$, each student in $N_{c}$ is assigned to $c$, and for each $t \in\{1,2\}$, each student in $N_{c_{t}}$ is assigned to $c_{t}$ and each student in $N_{c_{t}^{\prime}}^{\prime}$ is assigned to $c_{t}^{\prime}$.

\section{REFERENCES}

Abdulkadiroğlu, Atila, Yeon-Koo Che, Parag A. Pathak, Alvin E. Roth, and Olivier Tercieux (2020), "Efficiency, justified envy, and incentives in priority-based matching." American Economic Review Insights, 2, 425-442. [1251, 1252, 1271, 1272]

Abdulkadiroğlu, Atila, Yeon-Koo Che, and Yosuke Yasuda (2015), "Expanding "choice" in school choice.” American Economic Journal: Microeconomics, 7, 1-42. [1250]

Abdulkadiroğlu, Atila, Parag Pathak, and Alvin Roth (2009), "Strategy-proofness versus efficiency in matching with indifferences: Redesigning the NYC high school match." American Economic Review, 99, 1954-1978. [1250, 1271]

Abdulkadiroğlu, Atila and Tayfun Sönmez (2003), "School choice: A mechanism design approach.” American Economic Review, 93, 729-747. [1249, 1250, 1255, 1271]

Abraham, J., P. Biró, and D. F. Manlove (2005), "Almost stable matchings in the roommates problem." In Proceedings of WAOA 2005: The 3rd Workshop on Approximation and Online Algorithms, volume 3879, 1-14, Springer. [1252]

Andersson, Tommy, Lars Ehlers, and Lars-Gunnar Svensson (2014), "Budget-balance, fairness and minimal manipulability.” Theoretical Economics, 9, 753-777. [1252]

Bonkoungou, Somouaoga and Alexander Nesterov (2020), "Reforms meet fairness concerns in school and college admissions." Working paper, available at SSRN https://ssrn. com/abstract=3664089 or http://dx.doi.org/10.2139/ssrn.3664089. [1252]

Che, Yeon-Koo and Olivier Tercieux (2019), "Efficiency and stability in large matching markets." Journal of Political Economy, 127, 2301-2342. [1250]

Combe, Julien, Olivier Tercieux, and Camille Terrier (2017), "The design of teacher assignment: Theory and evidence.” Working paper. [1253]

Doğan, Battal, Serhat Doğan, and Kemal Yıldız (2018), "A new ex-ante efficiency criterion and implications for the probabilistic serial mechanism." Journal of Economic Theory, 175, 178-200. [1252] 
Doğan, Battal and Lars Ehlers (2020a), "Blocking pairs versus blocking students: Stability comparisons in school choice." Working paper, available at SSRN https://ssrn.com/ abstract=3571173 or http://dx.doi.org/10.2139/ssrn.3571173. [1249, 1267]

Doğan, Battal and Lars Ehlers (2020b), "Robust minimal instability of the top trading cycles mechanism.” Working paper. [1251, 1271]

Doğan, Battal and M. Bumin Yenmez (2020), "Consistent Pareto improvement over the student-optimal stable mechanism.” Economic Theory Bulletin, 8, 125-137. [1252, 1273]

Dur, Umut, A. Arda Gitmez, and Özgür Yılmaz (2019), "School choice under partial fairness.” Theoretical Economics, 14, 1309-1346. [1250, 1252, 1264, 1265]

Ehlers, Lars and Thayer Morrill (2020), “(Il)legal assignments in school choice.” The Review of Economic Studies, 87, 1837-1875. [1251, 1252]

Erdil, Aytek and Haluk Ergin (2008), "What's the matter with tie-breaking? Improving efficiency in school choice.” American Economic Review, 98, 669-689. [1250]

Ergin, Haluk I. (2002), "Efficient resource allocation on the basis of priorities.” Econometrica, 70, 2489-2497. [1252, 1256, 1262, 1263, 1265, 1266, 1275]

Gale, David and Lloyd S. Shapley (1962), "College admissions and the stability of marriage.” The American Mathematical Monthly, 69, 9-15. [1249, 1250, 1255]

Kesten, Onur (2010), “School choice with consent.” The Quarterly Journal of Economics, 125, 1297-1348. [1250, 1255, 1256, 1271, 1272]

Kesten, Onur and Morimitsu Kurino (2019), "Strategy-proof improvements upon deferred acceptance: A maximal domain for possibility." Games and Economic Behavior, 117, 120-143. [1250]

Kwon, Hyukjun and Ran I. Shorrer (2019), "Justified-envy minimal mechanisms in school choice.” Working paper, available at SSRN https://ssrn.com/abstract=3495266. [1252, 1272]

Maus, Stefan, Hans Peters, and Ton Storcken (2007a), "Minimal manipulability: Anonymity and unanimity." Social Choice and Welfare, 29, 247-269. [1252]

Maus, Stefan, Hans Peters, and Ton Storcken (2007b), "Anonymous voting and minimal manipulability." Journal of Economic Theory, 135, 533-544. [1252]

Pathak, Parag A. and Tayfun Sönmez (2013), "School admissions reform in Chicago and England: Comparing mechanisms by their vulnerability to manipulation." American Economic Review, 103, 80-106. [1252]

Romm, Assaf, Alvin E. Roth, and Ran I. Shorrer (2020), "Stability vs. no justified envy." Working paper, available at SSRN https://ssrn.com/abstract=3550122. [1272]

Roth, Alvin E. (1982), “The economics of matching: Stability and incentives.” Mathematics of Operations Research, 7, 617-628. [1250, 1253] 
Shapley, Lloyd and Herbert Scarf (1974), "On cores and indivisibility." Journal of Mathematical Economics, 1, 23-37. [1271, 1272]

Tang, Qianfeng and Jingsheng Yu (2014), “A new perspective on Kesten's school choice with consent idea." Journal of Economic Theory, 154, 543-561. [1255, 1256, 1273]

Tang, Qianfeng and Yongchao Zhang (2021), "Weak stability and Pareto efficiency in school choice.” Economic Theory, 71, 533-552. [1250, 1252, 1256]

Troyan, Peter, David Delacrétaz, and Andrew Kloosterman (2020), "Essentially stable matchings." Games and Economic Behavior, 120, 370-390. [1252]

Co-editor Federico Echenique handled this manuscript.

Manuscript received 9 April, 2020; final version accepted 23 January, 2021; available online 3 February, 2021. 